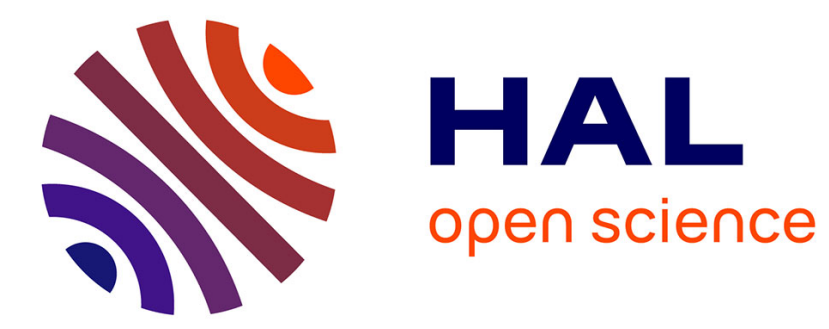

\title{
Simultaneous global exact controllability in projection of infinite 1D bilinear Schrödinger equations
}

\author{
Alessandro Duca
}

\section{To cite this version:}

Alessandro Duca. Simultaneous global exact controllability in projection of infinite 1D bilinear Schrödinger equations. Dynamics of Partial Differential Equations, 2020, 17 (3), pp.275-306. 10.4310/DPDE.2020.v17.n3.a4 . hal-01481873v5

\section{HAL Id: hal-01481873 \\ https://hal.science/hal-01481873v5}

Submitted on 16 Jul 2020

HAL is a multi-disciplinary open access archive for the deposit and dissemination of scientific research documents, whether they are published or not. The documents may come from teaching and research institutions in France or abroad, or from public or private research centers.
L'archive ouverte pluridisciplinaire HAL, est destinée au dépôt et à la diffusion de documents scientifiques de niveau recherche, publiés ou non, émanant des établissements d'enseignement et de recherche français ou étrangers, des laboratoires publics ou privés.

\section{다)(1) $(5$}

Distributed under a Creative Commons Attribution - NonCommerciall 4.0 International 


\title{
Simultaneous global exact controllability in projection of infinite 1D bilinear Schrödinger equations
}

\begin{abstract}
A. Duca
ABstract. The aim of this work is to study the controllability of infinite bilinear Schrödinger equations on a segment. We consider the equations (BSE) $i \partial_{t} \psi^{j}=-\Delta \psi^{j}+u(t) B \psi^{j}$ in the Hilbert space $L^{2}((0,1), \mathbb{C})$ for every $j \in$ $\mathbb{N}^{*}$. The Laplacian $-\Delta$ is equipped with Dirichlet homogeneous boundary conditions, $B$ is a bounded symmetric operator and $u \in L^{2}((0, T), \mathbb{R})$ with $T>$ 0 . We prove the simultaneous local and global exact controllability of infinite (BSE) in projection. The local controllability is guaranteed for any positive time and we provide explicit examples of $B$ for which our theory is valid. In addition, we show that the controllability of infinite (BSE) in projection onto suitable finite dimensional spaces is equivalent to the controllability of a finite number of (BSE) (without projecting). In conclusion, we rephrase our controllability results in terms of density matrices.
\end{abstract}

\section{Contents}

1. Introduction 1

2. Auxiliary results 5

3. Simultaneous local exact controllability in projection 8

4. Simultaneous global exact controllability in projection 14

5. Global exact controllability in projection for density matrices 20

6. Conclusion 22

Appendix A. Moment problem $\quad 23$

$\begin{array}{ll}\text { Appendix B. Analytic Perturbation } & 25\end{array}$

References 31

\section{Introduction}

1.1. The problem. In this work, we consider infinite particles constrained in a one-dimensional bounded region and subjected to an external control field. A

1991 Mathematics Subject Classification. Primary 93C20, 93B05; Secondary 35Q41, 81Q15.

Key words and phrases. Schrödinger equation, simultaneous control, global exact controllability, moment problem, perturbation theory, density matrices.

The author thanks Thomas Chambrion for suggesting him the problem and Nabile Boussaïd for the periodic discussions. He is also grateful to Morgan Morancey for the explanation about the works $[\mathbf{1 5}]$ and $[\mathbf{1 6}]$. 
suitable choice for such setting is to model the dynamics of these particles by infinitely many bilinear Schrödinger equations in the Hilbert space $\mathscr{H}=L^{2}((0,1), \mathbb{C})$

$$
\begin{cases}i \partial_{t} \psi_{j}(t)=A \psi_{j}(t)+u(t) B \psi_{j}(t), & t \in(0, T), T>0, \\ \psi_{j}(0)=\psi_{j}^{0} \in L^{2}((0,1), \mathbb{C}), & j \in \mathbb{N}^{*} .\end{cases}
$$

The Laplacian $A=-\Delta$ is equipped with homogeneous Dirichlet boundary conditions such that

$$
D(A)=H^{2}((0,1), \mathbb{C}) \cap H_{0}^{1}((0,1), \mathbb{C}) .
$$

The bounded symmetric operator $B$ models the action of the external field, while the control function $u \in L^{2}((0, T), \mathbb{R})$ represents its intensity.

We study the controllability of the infinite bilinear Schrödinger equations (BSE) at the same time $T$, with one unique control $u$ and by projecting onto suitable finite dimensional subspaces of $\mathscr{H}$.

In order to detail the purpose of the work, we introduce the following notations. We denote by $\Gamma_{t}^{u}$ the unitary propagator in $\mathscr{H}$ generated by the dynamics of the (BSE) in a time interval $[0, t]$ (when it is defined). Let $\Psi:=\left(\psi_{j}\right)_{j \in \mathbb{N}^{*}}$ an orthonormal system of $\mathscr{H}$. We call $\pi_{N}(\Psi)$ with $N \in \mathbb{N}^{*}$ the orthogonal projector

$$
\pi_{N}(\Psi): \mathscr{H} \longrightarrow \operatorname{span}\left\{\psi_{k}: k \leq N\right\} .
$$

We say that two sequences of functions $\left(\psi_{j}^{1}\right)_{j \in \mathbb{N}^{*}},\left(\psi_{j}^{2}\right)_{j \in \mathbb{N}^{*}} \subseteq \mathscr{H}$ are unitarily equivalent when there exists $\Gamma \in U(\mathscr{H})$ (the space of the unitary operators in $\mathscr{H}$ ) such that

$$
\psi_{j}^{1}=\Gamma \psi_{j}^{2}, \quad \forall j \in \mathbb{N}^{*} .
$$

The aim of this work is to study the existence of orthonormal systems $\Psi$ of $\mathscr{H}$ so that, for any $N \in \mathbb{N}^{*}$ and for any suitable $\left(\psi_{j}^{1}\right)_{j \in \mathbb{N}^{*}}$ and $\left(\psi_{j}^{2}\right)_{j \in \mathbb{N}^{*}}$ unitarily equivalent, there exist $T>0$ and $u \in L^{2}((0, T), \mathbb{R})$ such that

$$
\pi_{N}(\Psi) \Gamma_{T}^{u} \psi_{j}^{1}=\pi_{N}(\Psi) \psi_{j}^{2}, \quad \forall j \in \mathbb{N}^{*} .
$$

If we denote by $\langle\cdot, \cdot\rangle_{L^{2}}$ the usual $L^{2}$-scalar product, then the identities (1.2) become

$$
\left\langle\psi_{k}, \Gamma_{T}^{u} \psi_{j}^{1}\right\rangle_{L^{2}}=\left\langle\psi_{k}, \psi_{j}^{2}\right\rangle_{L^{2}}, \quad \forall j, k \in \mathbb{N}^{*}, k \leq N .
$$

In order to achieve the result, we show that the simultaneous global exact controllability in projection onto suitable $N$ dimensional spaces is equivalent to the controllability of $N$ problems (BSE) (without projecting).

1.2. Main results. Let $\|\cdot\|_{L^{2}}$ be the norm of the Hilbert space $\mathscr{H}=L^{2}((0,1), \mathbb{C})$ and $\langle\cdot, \cdot\rangle_{L^{2}}$ be the corresponding scalar product. Let

$$
\left(\phi_{j}\right)_{j \in \mathbb{N}^{*}}, \quad\left(\lambda_{j}\right)_{j \in \mathbb{N}^{*}}
$$

respectively be the eigenfunctions and the eigenvalues of $A$ such that

$$
\phi_{j}(x)=\sqrt{2} \sin (j \pi x), \quad \lambda_{j}=\pi^{2} j^{2}, \quad \forall j \in \mathbb{N}^{*} .
$$

We notice that $\left(\phi_{j}\right)_{j \in \mathbb{N}^{*}}$ forms a complete orthonormal system of $\mathscr{H}$ and we consider the spaces

$$
\begin{gathered}
H_{(0)}^{3}:=D\left(|A|^{\frac{3}{2}}\right), \quad\|\cdot\|_{(3)}:=\|\cdot\|_{H_{(0)}^{3}}=\left(\sum_{k=1}^{\infty}\left|k^{3}\left\langle\cdot, \phi_{k}\right\rangle_{L^{2}}\right|^{2}\right)^{\frac{1}{2}}, \\
\ell^{\infty}\left(H_{(0)}^{3}\right)=\left\{\left(\psi_{j}\right)_{j \in \mathbb{N}^{*}} \subset H_{(0)}^{3} \mid \sup _{j \in \mathbb{N}^{*}}\left\|\psi_{j}\right\|_{(3)}<\infty\right\} .
\end{gathered}
$$


For $s \in \mathbb{N}^{*}$, we call $H^{s}:=H^{s}((0,1), \mathbb{C}), H_{0}^{1}:=H_{0}^{1}((0,1), \mathbb{C})$ and, for $N \in \mathbb{N}^{*}$, we define

$$
I^{N}:=\left\{(j, k) \in \mathbb{N}^{*} \times\{1, \ldots, N\}: j>k\right\} .
$$

Assumptions I. The operator $B$ is bounded and symmetric in the Hilbert space $\mathscr{H}=L^{2}((0,1), \mathbb{C})$. In addition, it satisfies the following conditions.

(1) For any $N \in \mathbb{N}^{*}$, there exists $C_{N}>0$ such that $\left|\left\langle\phi_{k}, B \phi_{j}\right\rangle_{L^{2}}\right| \geq \frac{C_{N}}{k^{3}}$ for every $j, k \in \mathbb{N}^{*}$ with $j \leq N$.

(2) $\operatorname{Ran}\left(\left.B\right|_{H_{(0)}^{2}}\right) \subseteq H_{(0)}^{2}$ and $\operatorname{Ran}\left(\left.B\right|_{H_{(0)}^{3}}\right) \subseteq H^{3} \cap H_{0}^{1}$.

(3) For every $N \in \mathbb{N}^{*}$ and for every $(j, k),(l, m) \in I^{N}$ such that $(j, k) \neq(l, m)$ and $j^{2}-k^{2}-l^{2}+m^{2}=0$, we have

$$
\left\langle\phi_{j}, B \phi_{j}\right\rangle_{L^{2}}-\left\langle\phi_{k}, B \phi_{k}\right\rangle_{L^{2}}-\left\langle\phi_{l}, B \phi_{l}\right\rangle_{L^{2}}+\left\langle\phi_{m}, B \phi_{m}\right\rangle_{L^{2}} \neq 0
$$

The first condition in Assumptions I quantifies how much $B$ mixes eigenstates, while the second fixes its regularity. The third condition instead is required in order to decouple, through perturbation theory techniques, the eigenvalues resonances appearing in the proof of the following statement.

The next theorem states one of the main results of the work that is the simultaneous global exact controllability in projection of infinite (BSE). In order to keep this introduction as simple as possible, we postpone to Section 3 the second important result of the work which is the simultaneous local exact controllability in projection for any positive time (Theorem 3.1).

TheOREM 1.1. Let $\Gamma_{t}^{u}$ be the unitary propagator in $\mathscr{H}$ generated by the $d y$ namics of the (BSE) in the time interval $[0, t]$ with $B$ satisfying Assumptions $I$. Assume that $\Psi:=\left(\psi_{j}\right)_{j \in \mathbb{N}^{*}} \subset H_{(0)}^{3}$ is an orthonormal system of $\mathscr{H}$. Let $\left(\psi_{j}^{1}\right)_{j \in \mathbb{N}^{*}}$ and $\left(\psi_{j}^{2}\right)_{j \in \mathbb{N}^{*}} \subset H_{(0)}^{3}$ be complete orthonormal systems of $\mathscr{H}$ and $\widehat{\Gamma} \in U(\mathscr{H})$ be the unitary operator such that $\left(\widehat{\Gamma} \psi_{j}^{2}\right)_{j \in \mathbb{N}^{*}}=\left(\psi_{j}^{1}\right)_{j \in \mathbb{N}^{*}}$. If the following condition is satisfied

$$
\left(\widehat{\Gamma} \psi_{j}\right)_{j \leq N} \subset H_{(0)}^{3}
$$

with $N \in \mathbb{N}^{*}$, then there exist $T>0, u \in L^{2}((0, T), \mathbb{R})$ and $\left(\theta_{k}\right)_{k \leq N} \subset \mathbb{R}$ such that

$$
\left\langle\psi_{k}, \Gamma_{T}^{u} \psi_{j}^{1}\right\rangle_{L^{2}}=e^{i \theta_{k}}\left\langle\psi_{k}, \psi_{j}^{2}\right\rangle_{L^{2}}, \quad \forall j, k \in \mathbb{N}^{*}, k \leq N .
$$

Theorem 1.1 allows to control with a single $u$ and at the same time $T$ any finite number of components of infinitely many solutions of the problems (BSE). We notice that the statement is ensured up to phases in the components which prevents to formulate the result in terms of projectors. In addition, the orthonormal system $\left(\psi_{j}\right)_{j \in \mathbb{N}^{*}}$ has to verify a $H_{(0)}^{3}$-compatibility condition exposed in (1.5). Despite this assumption may seem unusual, it spontaneously appears when we try to control in projection infinite (BSE). We provide further discussions on the subject in Remark 4.2 where we show that it is a natural constraint for this kind of problems.

When we want to control in projection with respect to the target orthonormal system by using Theorem 1.1 , we choose $\Psi \equiv \Psi^{2}$. In this case, we notice that

$$
\left(\widehat{\Gamma} \psi_{j}\right)_{j \leq N}=\left(\widehat{\Gamma} \psi_{j}^{2}\right)_{j \leq N}=\left(\psi_{j}^{1}\right)_{j \leq N} \subset H_{(0)}^{3}
$$

and the $H_{(0)}^{3}$-compatibility condition (1.5) is trivially satisfied. In addition,

$$
e^{i \theta_{k}}\left\langle\psi_{k}^{2}, \psi_{j}^{2}\right\rangle_{L^{2}}=e^{i \theta_{k}} \delta_{k, j}=e^{i \theta_{j}}\left\langle\psi_{k}^{2}, \psi_{j}^{2}\right\rangle_{L^{2}}, \quad \forall j, k \in \mathbb{N}^{*} .
$$


Thus, the relations (1.6) become

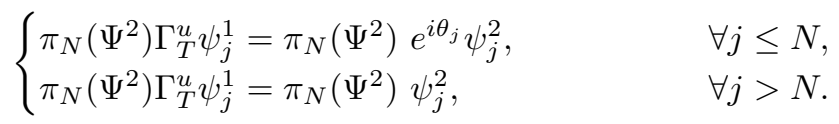

As $\Psi^{2}$ is composed by orthonormal elements, the projector appearing in the first line of (1.7) acts as the identity operator and the right-hand side of the second line is equal to 0 . These facts lead to the following corollary.

COROllary 1.2. Let $\Gamma_{t}^{u}$ be the unitary propagator in $\mathscr{H}$ generated by the dynamics of the (BSE) in the time interval $[0, t]$ with $B$ satisfying Assumptions $I$. Let $\Psi^{1}:=\left(\psi_{j}^{1}\right)_{j \in \mathbb{N}^{*}}, \Psi^{2}:=\left(\psi_{j}^{2}\right)_{j \in \mathbb{N}^{*}} \subset H_{(0)}^{3}$ be complete orthonormal systems of $\mathscr{H}$. For every $N \in \mathbb{N}^{*}$, there exist $T>0, u \in L^{2}((0, T), \mathbb{R})$ and $\left(\theta_{j}\right)_{j \leq N} \subset \mathbb{R}$ such that

$$
\begin{cases}\Gamma_{T}^{u} \psi_{j}^{1}=e^{i \theta_{j}} \psi_{j}^{2}, & \forall j \leq N, \\ \pi_{N}\left(\Psi^{2}\right) \Gamma_{T}^{u} \psi_{j}^{1}=0, & \forall j>N .\end{cases}
$$

Here, one can notice the parallelism between our results with the ones provided in the important work [16] by Morancey and Nersesyan. Indeed, Corollary 1.2 implies the controllability of any finite number of bilinear Schrödinger equations when $B$ satisfies Assumptions I. Similar results are provided in [16] and here we rephrase the main one.

TheOREM 1.3. [16, Main Theorem] Let the bilinear Schrödinger equation (BSE) be considered with $B=M_{\mu}$ a multiplication operator for a function $\mu \in H^{4}$. Fixed $N \in \mathbb{N}^{*}$, there exists $\mathcal{Q}$ a residual set of $H^{4}$ (an intersection of countably many subsets of $H^{4}$ with dense interiors) such that, for every $B=M_{\mu}$ with $\mu \in \mathcal{Q}$, the following result is satisfied. For any $\left(\psi_{k}^{1}\right)_{k \leq N},\left(\psi_{k}^{2}\right)_{k \leq N} \subset H_{(0)}^{3}$ unitarily equivalent, there exist $T>0$ and $u \in L^{2}((0, T), \mathbb{R})$ such that $\Gamma_{T}^{u} \psi_{k}^{1}=\psi_{k}^{2}$ for every $k \leq N$.

As we show in Section 4.2, the controllability of infinite (BSE) in projection is equivalent to the controllability of a finite number of (BSE) (without projecting). In view of this fact, similar statements to Theorem 1.1 can be provided by using the theory developed in Section 4.2 and the one from [16]. Even though such results can be really interesting, they are ensured with respect to abstract control operators $B$ (of multiplicative type) and then the controllability is only generically verified. From this perspective, our purpose is different. We aim to ensure the simultaneous global exact controllability when simple and explicit hypotheses on the problem, such as Assumptions I, are satisfied. This fact allows us to provide examples of $B$ for which the result is guaranteed, i.e. $B: \psi \in \mathscr{H} \mapsto x^{2} \psi$ (we refer to Example 2.2 for further details on this case and for other examples). Our goal is achieved by using different techniques from $[\mathbf{1 6}]$ whose disadvantage is the loss of control on the phase terms appearing in Theorem 1.1 and Corollary 1.2.

The other main contributions of the work are the following. First, we prove the equivalence between the controllability of infinitely many (BSE) in projection and the controllability of a finite number of equations without projection. Second, we prove the local controllability in any positive time $T>0$ which is stated in Section 3. Third, we use Theorem 1.1 and Corollary 1.2 in order to ensure the global exact controllability in projection for density matrices in Section 5 . 
1.3. A brief bibliography. Global approximate controllability results for the bilinear Schrödinger equation are provided with different techniques in literature. For instance, adiabatic arguments are considered by Boscain, Chittaro, Gauthier, Mason, Rossi and Sigalotti in [6] and [7]. The controllability is achieved with Lyapunov techniques by Mirrahimi in [14] and by Nersesyan in [17]. Lie-Galerkin arguments are used by Boscain, Boussaï, Caponigro, Chambrion, Mason and Sigalotti in $[\mathbf{5}],[\mathbf{8}]$ and $[\mathbf{1 0}]$.

The exact controllability of the bilinear Schrödinger equation (BSE) is in general a more delicate matter as a consequence of the results provided in the work on bilinear systems $[\mathbf{2}]$ by Ball, Mardsen and Slemrod. There, they prove that the (BSE) is not exactly controllable in the Hilbert space where it is defined when $B$ is a bounded operator and $u \in L_{\text {loc }}^{2}\left(\mathbb{R}^{+}, \mathbb{R}\right)$ (even though it is well-posed).

Despite this non-controllability result, many authors have addressed the problem for weaker notions of controllability by considering suitable subspaces of $D(A)$. This idea was preliminarily introduced by Beauchard in $[\mathbf{3}]$ and popularized by the work in [4]. In [4], Beauchard and Laurent prove the local exact controllability of (BSE) in a neighborhood of the first eigenfunction of $A$ in $S \cap H_{(0)}^{3}$ when $B$ is a suitable multiplication operator. The same kind of operators are considered in [15], where Morancey ensures the simultaneous local exact controllability in $S \cap H_{(0)}^{3}$ for at most three problems (BSE) and up to phases. In the work [16], Morancey and Nersesyan extend such result and prove Theorem 1.3.

1.4. Scheme of the work. In Section 2, we fix the notations considered in the work and we present some preliminary features of the problem such as the well-posedness of the (BSE) in the space $H_{(0)}^{3}$ proved in [4].

In Section 3, we ensure Theorem 3.1 which states the simultaneous local exact controllability in projection for any positive time up to phases. In order to motivate the modification of the problem, we emphasize the obstructions to overcome.

In Section 4, we prove Theorem 1.1. First, we show that the simultaneous global exact controllability in projection is equivalent to the controllability of finite (BSE) in Proposition 4.1. Second, we ensure with Proposition 4.5 the simultaneous global exact controllability of finite (BSE) by using the theory from Section 3 and a global approximate controllability. The propositions 4.1 and 4.5 lead to Theorem 1.1.

In Section 5, we rephrase our results in terms of density matrices, while in Section 6 , we provide some conclusive comments on the work.

In Appendix A, we briefly discuss the solvability of the so-called moment problems, while in Appendix B, we develop the perturbation theory techniques adopted in the work.

\section{Auxiliary results}

2.1. Notations and preliminaries. We denote by $\mathscr{H}$ the Hilbert space $L^{2}((0,1), \mathbb{C})$ equipped with the norm $\|\cdot\|_{L^{2}}$ and the scalar product $\langle\cdot, \cdot\rangle_{L^{2}}$ such that

$$
\langle f, g\rangle_{L^{2}}=\int_{0}^{1} \bar{f}(x) g(x) d x, \quad \forall f, g \in \mathscr{H} .
$$


Let $\mathscr{B}$ be a Banach space. We introduce for $s>0$,

$$
\begin{gathered}
H_{(0)}^{s}=D\left(|A|^{\frac{s}{2}}\right), \quad\|\cdot\|_{(s)}=\|\cdot\|_{H_{(0)}^{s}}=\left(\sum_{k=1}^{\infty}\left|k^{s}\left\langle\cdot, \phi_{k}\right\rangle_{L^{2}}\right|^{2}\right)^{\frac{1}{2}}, \\
h^{s}(\mathscr{B})=\left\{\left(\psi_{j}\right)_{j \in \mathbb{N}^{*}} \subset \mathscr{B} \mid \sum_{j=1}^{\infty}\left(j^{s}\left\|\psi_{j}\right\|_{\mathscr{B}}\right)^{2}<\infty\right\}, \\
\ell^{\infty}(\mathscr{B})=\left\{\left(\psi_{j}\right)_{j \in \mathbb{N}^{*}} \subset \mathscr{B} \mid \sup _{j \in \mathbb{N}^{*}}\left\|\psi_{j}\right\|_{\mathscr{B}}<\infty\right\} .
\end{gathered}
$$

We recall that $\left(\phi_{j}\right)_{j \in \mathbb{N}^{*}}$ is a complete orthonormal system of $\mathscr{H}$ composed by eigenfunctions of $A$ defined in (1.3) and related to the eigenvalues $\left(\lambda_{j}\right)_{j \in \mathbb{N}^{*}}$. Fixed

$$
\Psi:=\left(\psi_{j}\right)_{j \in \mathbb{N}^{*}} \subset \mathscr{H}, \quad \mathscr{H}_{N}(\Psi):=\operatorname{span}\left\{\psi_{j}: j \leq N\right\},
$$

we define $\pi_{N}(\Psi)$ the orthogonal projector such that

$$
\pi_{N}(\Psi): \mathscr{H} \longrightarrow \mathscr{H}_{N}(\Psi)
$$

REMARK 2.1. If a bounded operator $B$ satisfies Assumptions I, then $B \in$ $L\left(H_{(0)}^{2}, H_{(0)}^{2}\right)$. Indeed, $B$ is closed in $\mathscr{H}$, so for every $\left(u_{n}\right)_{n \in \mathbb{N}^{*}} \subset \mathscr{H}$ such that $u_{n} \stackrel{\mathscr{H}}{\longrightarrow} u$ and $B u_{n} \stackrel{\mathscr{H}}{\longrightarrow} v$, we have $B u=v$. Now, for every $\left(u_{n}\right)_{n \in \mathbb{N}^{*}} \subset H_{(0)}^{2}$ such that $u_{n} \stackrel{H_{(0)}^{2}}{\longrightarrow} u$ and $B u_{n} \stackrel{H_{(0)}^{2}}{\longrightarrow} v$, the convergences with respect to the $\mathscr{H}$-norm are implied and $B u=v$. Hence, the operator $B$ is closed in $H_{(0)}^{2}$ and $B \in L\left(H_{(0)}^{2}, H_{(0)}^{2}\right)$. The same argument leads to $B \in L\left(H_{(0)}^{3}, H^{3} \cap H_{0}^{1}\right)$ since $\operatorname{Ran}\left(\left.B\right|_{H_{(0)}^{3}}\right) \subseteq H^{3} \cap H_{0}^{1}$.

EXAmple 2.2. Assumptions I are satisfied for $B: \psi \mapsto x^{2} \psi$. Indeed, the condition 1) is guaranteed as

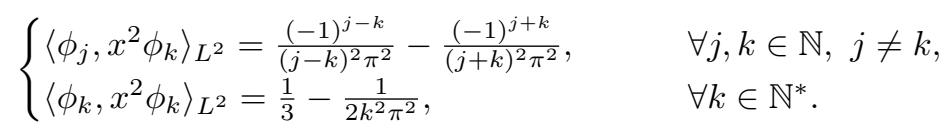

The point 2) of Assumptions I is trivially true, while the condition 3) is due to the following implication. For every $N \in \mathbb{N}^{*}$ and $(j, k),(l, m) \in I^{N}$ such that $(j, k) \neq(l, m)$ and $j^{2}-k^{2}-l^{2}+m^{2}=0$, we have

$$
j^{-2}-k^{-2}-l^{-2}+m^{-2} \neq 0 .
$$

We notice that the same properties are valid for other control operators. For instance, if we consider $B: \psi \in \mathscr{H} \longmapsto \sin \left(\frac{\pi}{2} x\right) \psi$, then Assumptions I are satisfied thanks to the identities

$$
\begin{cases}\left\langle\phi_{j}, B \phi_{k}\right\rangle_{L^{2}}=-\frac{32 j k}{\pi\left(16 j^{4}+16 k^{4}-8 j^{2}-8 k^{2}-32 k^{2} j^{2}+1\right)} & \forall j, k \in \mathbb{N}, j \neq k, \\ \left\langle\phi_{k}, B \phi_{k}\right\rangle_{L^{2}}=\frac{2}{\pi}+\frac{2}{\left(16 k^{2}-1\right) \pi}, & \forall k \in \mathbb{N}^{*} .\end{cases}
$$

The same is true for the operator $B: \psi \in \mathscr{H} \longmapsto x^{3} \psi$. Finally, an example of operator $B$ satisfying Assumptions I which is not of multiplicative type is

$$
B: \psi \in \mathscr{H} \longmapsto \sum_{j \in \mathbb{N}^{*}} \phi_{2 j-1}\left\langle\phi_{2 j-1}, x^{2} \psi\right\rangle+\sum_{j \in \mathbb{N}^{*}} \phi_{2 j}\left\langle\phi_{2 j}, \sin \left(\frac{\pi}{2} x\right) \psi\right\rangle \psi
$$


2.2. Well-posedness. In the current subsection, we cite an important result of well-posedness for the following problem in $\mathscr{H}$

$$
\left\{\begin{array}{l}
i \partial_{t} \psi(t)=A \psi(t)+u(t) \mu \psi(t), \quad t \in(0, T), \\
\psi(0)=\psi^{0} \in L^{2}((0,1), \mathbb{C}) .
\end{array}\right.
$$

Proposition 2.3. [4, Lemma 1; Proposition 2] Let $\mu \in H^{3}, T>0, \psi^{0} \in H_{(0)}^{3}$ and $u \in L^{2}((0, T), \mathbb{R})$. There exists a unique mild solution of $(2.3)$ in $H_{(0)}^{3}$, i.e. $\psi \in C^{0}\left([0, T], H_{(0)}^{3}\right)$ so that

$$
\psi(t)=e^{-i A t} \psi^{0}-i \int_{0}^{t} e^{-i A(t-s)} u(s) \mu \psi(s) d s, \quad \forall t \in[0, T] .
$$

Moreover, for every $R>0$, there exists $C=C(T, \mu, R)>0$ such that, if $\|u\|_{L^{2}((0, T), \mathbb{R})}<R$, then, for every $\psi^{0} \in H_{(0)}^{3}$, the solution satisfies

$$
\|\psi\|_{C^{0}\left([0, T], H_{(0)}^{3}\right)} \leq C\left\|\psi^{0}\right\|_{(3)}, \quad\|\psi(t)\|_{L^{2}}=\left\|\psi^{0}\right\|_{L^{2}}, \quad \forall t \in[0, T] .
$$

Remark 2.4. The result of Proposition 2.3 is not only valid for multiplication operators, but also for other suitable operators $B$. Indeed, the same proofs of $[4$, Lemma 1] and [4, Proposition 2] lead to the well-posedness of the (BSE) when $B$ is a bounded symmetric operator such that

$$
B \in L\left(H_{(0)}^{3}, H^{3} \cap H_{0}^{1}\right), \quad B \in L\left(H_{(0)}^{2}\right),
$$

which are verified if $B$ satisfies Assumptions I, thanks to Remark 2.1.

Let $\Gamma_{t}^{u}$ be the unitary propagator in $\mathscr{H}$ generated by the (BSE) in the time interval $[0, t]$. For any mild solution $\psi_{j}$ in $L^{2}((0,1), \mathbb{C})$ of the $j$-th problem (BSE) with $j \in \mathbb{N}^{*}$, we have

$$
\Gamma_{t}^{u} \psi_{j}(0)=\psi_{j}(t) .
$$

As a consequence of Remark 2.4, it follows $\left(\Gamma_{T}^{u} \psi_{j}\right)_{j \in \mathbb{N}^{*}} \in \ell^{\infty}\left(H_{(0)}^{3}\right)$ for every $\left(\psi_{j}\right)_{j \in \mathbb{N}^{*}} \in \ell^{\infty}\left(H_{(0)}^{3}\right)$. We refer to $(2.1)$ for the definition of the space $\ell^{\infty}\left(H_{(0)}^{3}\right)$.

2.3. Time reversibility. An important feature of the bilinear Schrödinger equation is the time reversibility. If we consider $\psi(t)=\Gamma_{t}^{u} \psi^{0}$ and we substitute $t$ with $T-t$ for $T>0$ in a bilinear Schrödinger equation, then we have

$$
\left\{\begin{array}{l}
i \partial_{t} \Gamma_{T-t}^{u} \psi^{0}=-A \Gamma_{T-t}^{u} \psi^{0}-u(T-t) B \Gamma_{T-t}^{u} \psi^{0}, \quad t \in(0, T), \\
\Gamma_{T-0}^{u} \psi^{0}=\Gamma_{T}^{u} \psi^{0}=\psi^{1}
\end{array}\right.
$$

We define the operator $\widetilde{\Gamma}_{t}^{\widetilde{u}}$ such that $\Gamma_{T-t}^{u} \psi^{0}=\widetilde{\Gamma}_{t}^{\widetilde{u}} \psi^{1}$ for $\widetilde{u}(t):=u(T-t)$ and

$$
\left\{\begin{array}{l}
i \partial_{t} \widetilde{\Gamma}_{t}^{\widetilde{u}} \psi^{1}=(-A-\widetilde{u}(t) B) \widetilde{\Gamma}_{t}^{\widetilde{u}} \psi^{1}, \quad t \in(0, T), \\
\widetilde{\Gamma}_{0}^{\widetilde{u}} \psi^{1}=\psi^{1} \in L^{2}((0,1), \mathbb{C}) .
\end{array}\right.
$$

As $\psi^{0}=\widetilde{\Gamma}_{T}^{\widetilde{u}} \Gamma_{T}^{u} \psi^{0}$ and $\psi^{1}=\Gamma_{T}^{u} \widetilde{\Gamma}_{T}^{\widetilde{u}} \psi^{1}$, it follows $\widetilde{\Gamma}_{T}^{\widetilde{u}}=\left(\Gamma_{T}^{u}\right)^{-1}=\left(\Gamma_{T}^{u}\right)^{*}$. The operator $\widetilde{\Gamma}_{t}^{\widetilde{u}}$ describes the reversed dynamics of $\Gamma_{t}^{u}$ induced by the system (2.4) and generated by the Hamiltonian $(-A-\widetilde{u}(t) B)$. 


\section{Simultaneous local exact controllability in projection}

3.1. Main result. In this section, we examine the simultaneous local exact controllability in projection stated by the following theorem.

Theorem 3.1. Let $\Gamma_{t}^{u}$ be the unitary propagator in $\mathscr{H}$ generated by the $d y$ namics of the (BSE) in the time interval $[0, t]$ with $B$ satisfying Assumptions $I$. Let $N \in \mathbb{N}^{*}$. For every $T>0$, there exist an open set $O$ in $\ell^{\infty}\left(H_{(0)}^{3}\right)$ and an orthonormal system $\Psi:=\left(\psi_{j}\right)_{j \in \mathbb{N}^{*}} \in O$ such that the following result is verified. Let $\left(\psi_{j}^{1}\right)_{j \in \mathbb{N}^{*}} \in O$ be a complete orthonormal system and $\widehat{\Gamma} \in U(\mathscr{H})$ be such that $\left(\widehat{\Gamma} \psi_{j}^{1}\right)_{j \in \mathbb{N}^{*}}=\left(\psi_{j}\right)_{j \in \mathbb{N}^{*}}$. If $\left(\widehat{\Gamma} \psi_{j}\right)_{j \leq N} \subset H_{(0)}^{3}$, then there exist $\left(\theta_{j}\right)_{j \leq N} \subset \mathbb{R}$ and $u \in L^{2}((0, T), \mathbb{R})$ such that

$$
\begin{cases}\left\langle\psi_{k}, \Gamma_{T}^{u} \psi_{j}\right\rangle_{L^{2}}=e^{i \theta_{j}}\left\langle\psi_{k}, \psi_{j}^{1}\right\rangle_{L^{2}}, & \forall j, k \in \mathbb{N}^{*}, j \leq N, k \leq N, \\ \left\langle\psi_{k}, \Gamma_{T}^{u} \psi_{j}\right\rangle_{L^{2}}=\left\langle\psi_{k}, \psi_{j}^{1}\right\rangle_{L^{2}}, & \forall j, k \in \mathbb{N}^{*}, j>N, k \leq N .\end{cases}
$$

In other words, the following identities are satisfied (with $\pi_{N}(\Psi)$ defined in (1.1)):

$$
\begin{cases}\pi_{N}(\Psi) \Gamma_{T}^{u} \psi_{j}=e^{i \theta_{j}} \pi_{N}(\Psi) \psi_{j}^{1}, & \forall j \in \mathbb{N}^{*}, j \leq N, \\ \pi_{N}(\Psi) \Gamma_{T}^{u} \psi_{j}=\pi_{N}(\Psi) \psi_{j}^{1}, & \forall j \in \mathbb{N}^{*}, j>N .\end{cases}
$$

3.2. Introductive discussion. We start by explaining why we need to modify the problem in order to prove Theorem 3.1. Let $\Phi=\left(\phi_{j}\right)_{j \in \mathbb{N}^{*}}$ be a complete orthonormal system composed by eigenfunctions of $A$. For every $j \in \mathbb{N}^{*}$, we denote $\phi_{j}(t, x)=e^{-i \lambda_{j} t} \phi_{j}(x)$ with $t>0$. From now on, we adopt the notation $\phi_{j}(t)=\phi_{j}(t, \cdot)$. Let $\epsilon>0$ and $T>0$. We consider the set

$$
\begin{aligned}
& O_{\epsilon, T}:=\{\left(\psi_{j}\right)_{j \in \mathbb{N}^{*}} \in \ell^{\infty}\left(H_{(0)}^{3}\right) \text { complete orthonormal system of } \mathscr{H} \text { such } \\
&\text { that } \left.\sup _{k \leq N} \sum_{j \in \mathbb{N}^{*}} k^{6}\left|\left\langle\psi_{j}, \phi_{k}(T)\right\rangle_{L^{2}}-\left\langle\phi_{j}(T), \phi_{k}(T)\right\rangle_{L^{2}}\right|^{2}<\epsilon\right\} .
\end{aligned}
$$

We would like to prove to validity of Theorem 3.1 in the neighborhood $O_{\epsilon, T}$ for $\epsilon, T>0$ with respect to the projector $\pi_{N}(\Phi)$ (see the definition (2.2)). Then,

$$
\Gamma_{T}^{u} \phi_{j}=\sum_{k=1}^{\infty} \phi_{k}(T)\left\langle\phi_{k}(T), \Gamma_{t}^{u} \phi_{j}\right\rangle_{L^{2}}, \quad \phi_{j}(T)=e^{-i \lambda_{j} T} \phi_{j}, \quad \forall j \in \mathbb{N}^{*}
$$

is the solution of the j-th (BSE) with initial data $\phi_{j}$ at time $T>0$. We consider the infinite matrix $\alpha(u)$ such that

$$
\alpha_{k, j}(u)=\left\langle\phi_{k}(T), \Gamma_{T}^{u} \phi_{j}\right\rangle_{L^{2}}, \quad \forall k, j \in \mathbb{N}^{*}, k \leq N .
$$

We would like to ensure the existence of $\epsilon>0$ and $T>0$ such that for any $\left(\psi_{j}\right)_{j \in \mathbb{N}^{*}} \in O_{\epsilon, T}$, there exists $u \in L^{2}((0, T), \mathbb{R})$ such that

$$
\pi_{N}(\Phi) \Gamma_{T}^{u} \phi_{j}=\pi_{N}(\Phi) \psi_{j}, \quad \forall j \in \mathbb{N}^{*} .
$$

This result can be proved by studying the local surjectivity of $\alpha$ for $T>0$. To this purpose, we want to use the inverse mapping theorem and study the surjectivity of 
the Fréchet derivative of $\alpha$ the infinite matrix $\gamma(v):=\left(d_{u} \alpha(0)\right) \cdot v$ such that

$$
\begin{aligned}
\gamma_{k, j}(v): & =\left\langle\phi_{k}(T),-i \int_{0}^{T} e^{-i A(T-s)} v(s) B e^{-i A s} \phi_{j} d s\right\rangle_{L^{2}} \\
& =-i \int_{0}^{T} v(s) e^{-i\left(\lambda_{j}-\lambda_{k}\right) s} d s B_{k, j}, \quad \forall j, k \in \mathbb{N}^{*}, k \leq N,
\end{aligned}
$$

with $B_{k, j}=\left\langle\phi_{k}, B \phi_{j}\right\rangle_{L^{2}}=\left\langle B \phi_{k}, \phi_{j}\right\rangle_{L^{2}}=\overline{B_{j, k}}$. The surjectivity of $\gamma$ consists in proving the solvability of the moment problem

$$
\frac{x_{k, j}}{B_{k, j}}=-i \int_{0}^{T} u(s) e^{-i\left(\lambda_{j}-\lambda_{k}\right) s} d s, \quad \forall j, k \in \mathbb{N}^{*}, k \leq N,
$$

for each infinite matrix $\mathbf{x}:=\left(x_{k, j}\right)_{\substack{j, k \in \mathbb{N}^{*} \\ k \leq N}}$ belonging to a suitable space. To this end, one would use Corollary A.9 which is consequence of the Haraux's Theorem but an obstruction appears. The terms $\left(\lambda_{j}-\lambda_{k}\right)_{\substack{j, k \in \mathbb{N}^{*} \\ k \leq N}}$ in the moment problem (3.2) present the so-called eigenvalues resonances. Formally, for some $j, k, n, m \in \mathbb{N}^{*}$ such that $j \neq k, n \neq m,(j, k) \neq(n, m)$ and $k, m \leq N$, there holds $\lambda_{j}-\lambda_{k}=\lambda_{n}-\lambda_{m}$, which implies

$$
\frac{x_{k, j}}{B_{k, j}}=-i \int_{0}^{T} u(s) e^{-i\left(\lambda_{j}-\lambda_{k}\right) s} d s=-i \int_{0}^{T} u(s) e^{-i\left(\lambda_{n}-\lambda_{m}\right) s} d s=\frac{x_{n, m}}{B_{n, m}} .
$$

An example of eigenvalues resonance is $\lambda_{7}-\lambda_{1}=\lambda_{8}-\lambda_{4}$, but many others can be listed. For instance, all the diagonal terms of $\gamma$ since $\lambda_{j}-\lambda_{k}=0$ for $j=k$. The relation (3.3) represents a constraint on the considered matrices $\mathbf{x}$ which is not naturally satisfied in our framework.

In order to avoid this phenomenon, we adopt the following strategy. First, we consider the Hamiltonian characterizing the bilinear Schrödinger equations (BSE) and we use the following decomposition

$$
A+u(t) B=\left(A+u_{0} B\right)+u_{1}(t) B, \quad u_{0} \in \mathbb{R}, u_{1} \in L^{2}((0, T), \mathbb{R}) .
$$

Second, we consider $A+u_{0} B$ instead of $A$. We repeat the previous steps by considering $\left(\phi_{j}^{u_{0}}\right)_{j \in \mathbb{N}^{*}}$ a complete orthonormal system of $\mathscr{H}$ composed by eigenfunctions of $A+u_{0} B$ and $\left(\lambda_{j}^{u_{0}}\right)_{j \in \mathbb{N}^{*}}$ the corresponding eigenvalues. By using $u_{0} B$ as a perturbation in $A+u_{0} B$, we modify the eigenvalues gaps

$$
\lambda_{j}^{u_{0}}-\lambda_{k}^{u_{0}}, \quad \forall j, k \in \mathbb{N}^{*}, k \leq N
$$

in order to remove all the non-diagonal resonances. Afterwards, we consider $\widehat{\alpha}$ depending on the parameter $u_{0}$ (instead of $\alpha$ ) such that it is defined by the elements $\widehat{\alpha}_{k, j}(u)=\left\langle e^{-i \lambda_{k}^{u_{0}} T} \phi_{k}^{u_{0}}, \Gamma_{T}^{u} \phi_{j}^{u_{0}}\right\rangle_{L^{2}}$ with $k, j \in \mathbb{N}^{*}$ and $k \leq N$. Now, we rotate the terms of $\widehat{\alpha}$ in order to remove the resonances on the diagonal terms. We denote by $\alpha^{u_{0}}$ the obtained map, which is defined by the elements

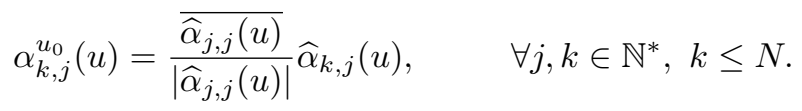

In conclusion, we use the inverse mapping theorem with respect to the map $\alpha^{u_{0}}$.

The first step of our strategy is not so different from the techniques leading to [16, Main Theorem], however it presents an important difference. In our work, we seek for explicit conditions on the operator $B$ such that the perturbative argument is valid. On the contrary, the authors of $[\mathbf{1 6}]$ prove the existence of $\mathcal{Q}$, a residual 
subset of $H^{4}((0,1), \mathbb{R})$ (in the spirit of Theorem 1.3), such that the controllability holds when $B$ is a multiplication operator by a function $\mu \in \mathcal{Q}$.

3.3. The modified problem. In this subsection, we rewrite the (BSE) by applying the decomposition (3.4) and we introduce the groundwork required to apply the strategy discussed in Section 3.2. Let $u(t)=u_{0}+u_{1}(t)$ with $u_{0} \in \mathbb{R}$, $u_{1} \in L^{2}((0, T), \mathbb{R})$ and $T>0$. We consider the following Cauchy problems

$$
\begin{cases}i \partial_{t} \psi_{j}(t)=\left(A+u_{0} B\right) \psi_{j}(t)+u_{1}(t) B \psi_{j}(t), & t \in(0, T), \\ \psi_{j}^{0}=\psi_{j}(0), & j \in \mathbb{N}^{*} .\end{cases}
$$

As $B$ is bounded, $A+u_{0} B$ has pure discrete spectrum. We recall that $\left(\lambda_{j}^{u_{0}}\right)_{j \in \mathbb{N}^{*}}$ are the eigenvalues of $A+u_{0} B$ and $\Phi^{u_{0}}:=\left(\phi_{j}^{u_{0}}\right)_{j \in \mathbb{N}^{*}}$ is a complete orthonormal system of $\mathscr{H}$ made by corresponding eigenfunctions. Fixed $N \in \mathbb{N}^{*}$, for every $T>0$ and $\epsilon_{0}>0$, we denote

$$
\begin{aligned}
O_{\epsilon_{0}, T}^{u_{0}}:= & \left\{\left(\psi_{j}\right)_{j \in \mathbb{N}^{*}} \in \ell^{\infty}\left(H_{(0)}^{3}\right) \text { complete orthonormal system of } \mathscr{H}\right. \text { such } \\
& \text { that } \left.\sup _{k \leq N} \sum_{j \in \mathbb{N}^{*}} k^{6}\left|\left\langle\psi_{j}, \phi_{k}^{u_{0}}(T)\right\rangle_{L^{2}}-\left\langle\phi_{j}^{u_{0}}(T), \phi_{k}^{u_{0}}(T)\right\rangle_{L^{2}}\right|^{2}<\epsilon_{0}\right\},
\end{aligned}
$$

with $\phi_{j}^{u_{0}}(T):=e^{-i \lambda_{j}^{u_{0}} T} \phi_{j}^{u_{0}}$. We choose $\left|u_{0}\right|$ small such that $\lambda_{k}^{u_{0}} \neq 0$ for every $k \in \mathbb{N}^{*}$ (Lemma B.4). The modification of the problem imposes to define the space

$$
\widetilde{H}_{(0)}^{3}:=D\left(\left|A+u_{0} B\right|^{\frac{3}{2}}\right), \quad\|\cdot\|_{\widetilde{H}_{(0)}^{3}}=\left(\left.\left.\sum_{k=1}^{\infty}|| \lambda_{k}^{u_{0}}\right|^{\frac{3}{2}}\left\langle\cdot, \phi_{k}^{u_{0}}\right\rangle_{L^{2}}\right|^{2}\right)^{\frac{1}{2}} .
$$

However, we consider from now on $u_{0}$ in the neighborhood provided by Lemma B.6 so that $\widetilde{H}_{(0)}^{3} \equiv H_{(0)}^{3}$. As introduced in Section 3.2, we consider the map $\widehat{\alpha}$ with elements $\widehat{\alpha}_{k, j}\left(u_{1}\right)=\left\langle\phi_{k}^{u_{0}}(T), \Gamma_{T}^{u_{0}+u_{1}} \phi_{j}^{u_{0}}\right\rangle_{L^{2}}$ for $k \leq N$ and $j \in \mathbb{N}^{*}$. The map $\alpha^{u_{0}}$ is the infinite matrix with elements

$$
\begin{cases}\alpha_{k, j}^{u_{0}}\left(u_{1}\right)=\frac{\overline{\hat{\alpha}_{j, j}\left(u_{1}\right)}}{\left|\hat{\alpha}_{j, j}\left(u_{1}\right)\right|} \widehat{\alpha}_{k, j}\left(u_{1}\right), & \forall j, k \in \mathbb{N}^{*}, j, k \leq N, \\ \alpha_{k, j}^{u_{0}}\left(u_{1}\right)=\widehat{\alpha}_{k, j}\left(u_{1}\right), & \forall j, k \in \mathbb{N}^{*}, j>N, k \leq N .\end{cases}
$$

Now, we study the space where $\alpha^{u_{0}}$ takes value. Let $\widetilde{\Gamma}_{t}^{\widetilde{u}}$ be the propagator of the reversed dynamics defined in Section 2.3 for $t \in[0, T], u \in L^{2}((0, T), \mathbb{R})$ and $T>0$. For every $k \in \mathbb{N}^{*}, u_{0} \in \mathbb{R}$ and $u_{1} \in L^{2}((0, T), \mathbb{R})$, from Proposition 2.3, Remark 2.4 and Lemma B.6, there exists $C>0$ so that

$$
\begin{aligned}
\sum_{j=1}^{+\infty} j^{6}\left|\alpha_{k, j}^{u_{0}}\left(u_{1}\right)\right|^{2} & =\sum_{j=1}^{+\infty} j^{6}\left|\left\langle\widetilde{\Gamma}_{T}^{u_{0}+\widetilde{u}_{1}} \phi_{k}^{u_{0}}, \phi_{j}^{u_{0}}\right\rangle_{L^{2}}\right|^{2}=\left\|\widetilde{\Gamma}_{T}^{u_{0}+\widetilde{u}_{1}} \phi_{k}^{u_{0}}\right\|_{\widetilde{H}_{(0)}^{3}}^{2} \\
& \leq C\left\|\widetilde{\Gamma}_{T}^{u_{0}+\widetilde{u}_{1}} \phi_{k}^{u_{0}}\right\|_{(3)}^{2}<\infty .
\end{aligned}
$$

Thus, each $\left(\alpha_{k, j}^{u_{0}}\left(u_{1}\right)\right)_{j \in \mathbb{N}^{*}} \in h^{3}(\mathbb{C})$ (defined in (2.1)). For every $\left(\psi_{j}\right)_{j \in \mathbb{N}^{*}}$ such that $\left(\psi_{j}\right)_{j \in \mathbb{N}^{*}} \in O_{\epsilon_{0}, T}^{u_{0}}$ or such that $\left(\psi_{j}\right)_{j \in \mathbb{N}^{*}}=\left(\Gamma_{T}^{u_{0}+u_{1}} \phi_{j}^{u_{0}}\right)_{j \in \mathbb{N}^{*}}$, we have

$$
\begin{aligned}
\delta_{j, k} & =\left\langle\phi_{j}^{u_{0}}, \phi_{k}^{u_{0}}\right\rangle_{L^{2}}=\left\langle\sum_{m \in \mathbb{N}^{*}} \psi_{m}\left\langle\psi_{m}, \phi_{j}^{u_{0}}\right\rangle_{L^{2}}, \sum_{l \in \mathbb{N}^{*}} \psi_{l}\left\langle\psi_{l}, \phi_{k}^{u_{0}}\right\rangle_{L^{2}}\right\rangle_{L^{2}} \\
& =\left\langle\left(\left\langle\psi_{m}, \phi_{j}^{u_{0}}\right\rangle_{L^{2}}\right)_{m \in \mathbb{N}^{*}},\left(\left\langle\psi_{m}, \phi_{k}^{u_{0}}\right\rangle_{L^{2}}\right)_{m \in \mathbb{N}^{*}}\right\rangle_{\ell^{2}}, \quad \forall j, k \leq N .
\end{aligned}
$$


The last relations imply that $\alpha^{u_{0}}: u \in L^{2}((0, T), \mathbb{R}) \longmapsto\left(\alpha_{k, j}^{u_{0}}(u)\right)_{\substack{k, j \in \mathbb{N}^{*} \\ k \leq N}} \in Q^{N}$ where

$$
\begin{aligned}
Q^{N}:= & \left\{\left(x_{k, j}\right)_{\substack{k, j \in \mathbb{N}^{*} \\
k \leq N}} \in\left(h^{3}(\mathbb{C})\right)^{N} \mid \quad x_{k, k} \in \mathbb{R},\right. \\
& \left.\left\langle\left(x_{j, l}\right)_{l \in \mathbb{N}^{*}},\left(x_{k, l}\right)_{l \in \mathbb{N}^{*}}\right\rangle_{\ell^{2}}=\delta_{j, k}, \quad \forall k, j \leq N\right\} .
\end{aligned}
$$

Now, $\Gamma_{T}^{(\cdot)} \psi: u \in L^{2}((0, T), \mathbb{R}) \longmapsto \Gamma_{T}^{u} \psi \in H_{(0)}^{3}$ with $\psi \in H_{(0)}^{3}$ is $C^{1}$ (see $[3$, Proposition 47] or [15, Section 2] for further details) and the same is true for $\widetilde{\Gamma}_{T}^{(\cdot)} \psi: u \in L^{2}((0, T), \mathbb{R}) \longmapsto \widetilde{\Gamma}_{T}^{u} \psi \in H_{(0)}^{3}$ for every $\psi \in H_{(0)}^{3}$. Finally, the map

$$
\alpha^{u_{0}}: u \in L^{2}((0, T), \mathbb{R}) \longmapsto\left(\alpha_{k, j}^{u_{0}}(u)\right)_{\substack{k, j \in \mathbb{N}^{*} \\ k \leq N}} \in Q^{N}
$$

is $C^{1}$ thanks to the identities $\widehat{\alpha}_{k, j}\left(u_{1}\right)=\left\langle\phi_{k}^{u_{0}}(T), \Gamma_{T}^{u_{0}+u_{1}} \phi_{j}^{u_{0}}\right\rangle=\left\langle\widetilde{\Gamma}_{T}^{u_{0}+, \widetilde{u}_{1}} \phi_{k}^{u_{0}}(T), \phi_{j}^{u_{0}}\right\rangle$ for every $k, j \in \mathbb{N}^{*}$ with $k \leq N$. We denote by $\gamma^{u_{0}}(v)=\left(\left(d_{u_{1}} \alpha^{u_{0}}\right)(0)\right) \cdot v$ the Fréchet derivative of $\alpha^{u_{0}}$. Defined $\widehat{\gamma}_{k, j}(v)=\left(\left(d_{u_{1}} \widehat{\alpha}\right)(0)\right) \cdot v$, the elements of $\gamma^{u_{0}}(v)$ are

$$
\begin{cases}\gamma_{k, j}^{u_{0}}=\left(\widehat{\gamma}_{j, j} \delta_{k, j}+\widehat{\gamma}_{k, j}-\delta_{k, j} \Re\left(\widehat{\gamma}_{j, j}\right)\right), & \forall j, k \in \mathbb{N}^{*}, j, k \leq N, \\ \gamma_{k, j}^{u_{0}}=\widehat{\gamma}_{k, j}, & \forall j, k \in \mathbb{N}^{*}, k \leq N, j>N\end{cases}
$$

and then, for $B_{k, j}^{u_{0}}=\left\langle\phi_{k}^{u_{0}}, B \phi_{j}^{u_{0}}\right\rangle_{L^{2}}$ for $k \leq N$ and $j \in \mathbb{N}^{*}$,

$$
\left\{\begin{array}{lll}
\gamma_{k, j}^{u_{0}}=\widehat{\gamma}_{k, j}=-i \int_{0}^{T} u_{1}(s) e^{-i\left(\lambda_{j}^{u_{0}}-\lambda_{k}^{u_{0}}\right) s} d s B_{k, j}^{u_{0},}, & & \forall j, k \in \mathbb{N}^{*}, k \neq j, \\
\gamma_{k, k}^{u_{0}}=\Re\left(\widehat{\gamma}_{k, k}\right)=0, & & \forall k \in \mathbb{N}^{*} .
\end{array}\right.
$$

The relation $\gamma_{k, k}^{u_{0}}=0$ is due to the fact that $\left(i \widehat{\gamma}_{k, k}\right) \in \mathbb{R}$ since $\widehat{\gamma}_{k, j}=-\bar{\gamma}_{j, k}$ for $j, k \leq N$. Hence, the diagonal elements of $\gamma^{u_{0}}$ are all equal to 0 due to the rotations adopted in the definition $\alpha^{u_{0}}$. Since $O_{\epsilon_{0}, T}^{u_{0}}$ is composed by orthonormal elements, the tangent space of $O_{\epsilon_{0}, T}^{u_{0}}$ in the point $\Phi^{u_{0}}$ is

$$
T_{\Phi^{u_{0}}} O_{\epsilon_{0}, T}^{u_{0}}=\left\{\left(\psi_{j}\right)_{j \in \mathbb{N}^{*}} \subset \ell^{\infty}\left(H_{(0)}^{3}\right) \mid\left\langle\phi_{k}^{u_{0}}, \psi_{j}\right\rangle_{L^{2}}=-\overline{\left\langle\phi_{j}^{u_{0}}, \psi_{k}\right\rangle_{L^{2}}}\right\} .
$$

The last relation implies that $\gamma^{u_{0}}: u \in L^{2}((0, T), \mathbb{R}) \longmapsto\left(\gamma_{k, j}^{u_{0}}(u)\right)_{\substack{k, j \in \mathbb{N}^{*} \\ k \leq N}} \in G^{N}$ where

$$
G^{N}:=\left\{\left(x_{k, j}\right)_{\substack{k, j \in \mathbb{N}^{*} \\ k \leq N}} \in\left(h^{3}(\mathbb{C})\right)^{N} \mid \quad x_{k, j}=-\overline{x_{j, k}}, \quad x_{k, k}=0, \quad \forall k, j \leq N\right\} .
$$

REMARK 3.2. When the third point of Remark B.9 is valid, the controllability in $O_{\epsilon_{0}, T}^{u_{0}}$ (defined in (3.6)) with $\epsilon_{0}>0$ ensures the controllability in $O_{\epsilon, T}$ (defined in (3.1)) for suitable $\epsilon>0$. Let $\left(\psi_{j}\right)_{j \in \mathbb{N}^{*}} \in O_{\epsilon, T}$ and $\widehat{\Gamma} \in U(\mathscr{H})$ be such that $\left(\widehat{\Gamma} \psi_{j}\right)_{j \in \mathbb{N}^{*}}=\left(\phi_{j}^{u_{0}}\right)_{j \in \mathbb{N}^{*}}$ and satisfying $\left(\widehat{\Gamma} \phi_{j}^{u_{0}}\right)_{j \leq N} \subset H_{(0)}^{3}$. There exists $C>0$ so that, for every $k \leq N$,

$$
\sum_{j \in \mathbb{N}^{*}}\left|j^{3}\left\langle\phi_{k}^{u_{0}}, \psi_{j}\right\rangle_{L^{2}}\right|^{2}=\sum_{j \in \mathbb{N}^{*}}\left|j^{3}\left\langle\widehat{\Gamma} \phi_{k}^{u_{0}}, \phi_{j}^{u_{0}}\right\rangle_{L^{2}}\right|^{2} \leq C\left\|\widehat{\Gamma} \phi_{k}^{u_{0}}\right\|_{(3)}<\infty
$$

thanks to Lemma B.4 and Lemma B.6. Now, fixed $e^{i \theta_{j}^{1}}:=\frac{\overline{\left\langle\phi_{j}^{u_{0}}(T), \psi_{j}\right\rangle_{L^{2}}}}{\left\langle\left\langle\phi_{j}^{u_{0}}(T), \psi_{j}\right\rangle_{L^{2}}\right|}$ for $j \leq N$ and $e^{i \theta_{j}^{1}}:=1$ for $j>N$, the relation (3.9) yields that $\left(e^{i \theta_{j}^{1}}\left\langle\phi_{k}^{u_{0}}(T), \psi_{j}\right\rangle_{L^{2}}\right)_{\substack{j, k \in \mathbb{N}^{*} \\ k \leq N}}$ 
belongs to $Q_{\epsilon}^{N}$ where

$$
Q_{\epsilon}^{N}:=\left\{\left(x_{k, j}\right)_{\substack{k, j \in \mathbb{N}^{*} \\ k \leq N}} \in Q^{N}\left|\sup _{k \leq N} \sum_{j \in \mathbb{N}^{*}} k^{6}\right| x_{k, j}-\left.\delta_{k, j}\right|^{2}<\epsilon\right\} .
$$

When $\alpha^{u_{0}}$ is surjective in $Q_{\epsilon}^{N}$, there exist $T>0$ and $u \in L^{2}((0, T), \mathbb{R})$ such that

$$
\begin{aligned}
& \left(e^{i \theta_{j}^{1}}\left\langle\phi_{k}^{u_{0}}, \psi_{j}\right\rangle_{L^{2}}\right)_{\substack{j, k \in \mathbb{N}^{*} \\
k \leq N}}=\left(e^{i \theta_{j}^{2}}\left\langle\phi_{k}^{u_{0}}, \Gamma_{T}^{u} \phi_{j}^{u_{0}}\right\rangle_{L^{2}}\right)_{\substack{j, k \in \mathbb{N}^{*} \\
k \leq N}}, \\
& \text { with } \begin{cases}e^{i \theta_{j}^{2}}:=\frac{\overline{\widehat{\alpha}_{j, j}\left(u_{1}\right)}}{\left|\widehat{\alpha}_{j, j}\left(u_{1}\right)\right|}, & j \leq N, \\
e^{i \theta_{j}^{2}:=1,} & j>N .\end{cases}
\end{aligned}
$$

Thus, the surjectivity of the map $\alpha^{u_{0}}$ in $Q_{\epsilon_{0}}^{N}$ ensures the validity of Theorem 3.1 with respect to the projector $\pi_{N}\left(\Phi^{u_{0}}\right)$ in $O_{\epsilon_{0}, T}^{u_{0}}$ and in $O_{\epsilon, T}$ for a suitable $\epsilon>0$.

3.4. Proof of Theorem 3.1. In the next proposition, we state the simultaneous local exact controllability in projection for any $T>0$ up to phases. The result implies Theorem 3.1 .

Proposition 3.3. Let $N \in \mathbb{N}^{*}$ and $B$ satisfy Assumptions I. For every $T>0$, there exist $\epsilon>0$ and $u_{0} \in \mathbb{R}$ such that the following result is verified. Let $\left(\psi_{j}^{1}\right)_{j \in \mathbb{N}^{*}} \in$ $O_{\epsilon, T}$ (defined in (3.1)) and $\widehat{\Gamma} \in U(\mathscr{H})$ be such that $\left(\widehat{\Gamma} \psi_{j}^{1}\right)_{j \in \mathbb{N}^{*}}=\left(\phi_{j}^{u_{0}}\right)_{j \in \mathbb{N}^{*}}$. If $\left(\widehat{\Gamma} \phi_{j}^{u_{0}}\right)_{j \leq N} \subset H_{(0)}^{3}$, then there exist $\left(\theta_{j}\right)_{j \leq N}$ and $u \in L^{2}((0, T), \mathbb{R})$ such that

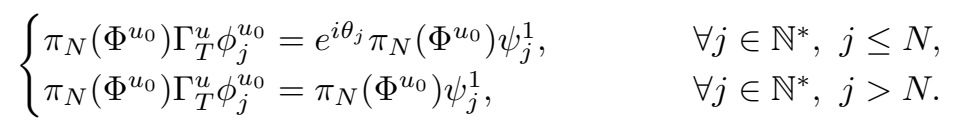

Proof. 1) Let $u_{0}$ belong to the neighborhoods defined in Appendix B by Lemma B.4, Lemma B.5, Lemma B.6 and Remark B.9. As discussed in Remark 3.2 , the surjectivity in $Q_{\epsilon}^{N}$ of the map $\alpha^{u_{0}}$ guarantees the simultaneous local exact controllability in projection up to phases in $O_{\epsilon, T}$.

We want to use the inverse mapping theorem by considering that $G^{N}$ is the tangent space of $Q^{N}$ in the point $\left(\delta_{k, j}\right)_{\substack{k, j \in \mathbb{N}^{*} \\ k \leq N}}=\alpha^{u_{0}}(0)$. If $\gamma^{u_{0}}$ is surjective in $G^{N}$ for $T>0$, then $\alpha^{u_{0}}$ is surjective in $Q_{\epsilon}^{N}$ for $\epsilon$ small enough. The surjectivity of $\gamma^{u_{0}}$ corresponds to the solvability of the moment problem

$$
x_{k, j}^{u_{0}} / B_{k, j}^{u_{0}}=-i \int_{0}^{T} u(s) e^{-i\left(\lambda_{j}^{u_{0}}-\lambda_{k}^{u_{0}}\right) s} d s, \quad \forall j, k \in \mathbb{N}^{*}, k \leq N
$$

for every $\left(x_{k, j}^{u_{0}}\right)_{\substack{j, k \in \mathbb{N}^{*} \\ k \leq N}} \in G^{N}$. We notice that the equations (3.11) for $k=j$ are redundant as $\gamma_{k, k}^{u_{0}}=0$ and $x_{k, k}^{u_{0}}=0$ for every $k \leq N$ since $\left(x_{k, j}^{u_{0}}\right)_{\substack{k, j \in \mathbb{N}^{*} \\ k \leq N}} \in G^{N}$. The same is true for $j, k \leq N$ such that $j<k$ since

$$
\left(x_{j, k}\right)_{j, k \leq N}, \quad\left(\gamma_{j, k}(u)\right)_{j, k \leq N} \quad \text { with } \quad u \in L^{2}((0, T), \mathbb{R}),
$$

are skew-hermitian matrices. Thus, we can prove the solvability of (3.11) for $k<j$ and $j=k=1$. Now, we have $\left(x_{k, j}^{u_{0}}\right)_{\substack{j, k \in \mathbb{N}^{*} \\ k \leq N}} \in\left(h^{3}\right)^{N}$ and $\left(\gamma_{k, j}^{u_{0}}\right)_{\substack{j, k \in \mathbb{N}^{*} \\ k \leq N}} \in\left(h^{3}\right)^{N}$. Lemma B.5 yields that

$$
\left(x_{k, j}^{u_{0}} / B_{k, j}^{u_{0}}\right)_{\substack{j, k \in \mathbb{N}^{*} \\ k \leq N}} \in\left(\ell^{2}(\mathbb{C})\right)^{N}, \quad\left(\gamma_{k, j}^{u_{0}} / B_{k, j}^{u_{0}}\right)_{\substack{j, k \in \mathbb{N}^{*} \\ k \leq N}} \in\left(\ell^{2}(\mathbb{C})\right)^{N} .
$$


Thanks to Lemma B.8, for $I^{N}$ defined in (1.4), there exists

$$
\begin{aligned}
\mathscr{G}^{\prime} & :=\sup _{A \subset I^{N}}\left(\inf _{\substack{(j, k),(n, m) \in I^{N} \backslash A \\
(j, k) \neq(n, m)}}\left|\lambda_{j}^{u_{0}}-\lambda_{k}^{u_{0}}-\lambda_{n}^{u_{0}}+\lambda_{m}^{u_{0}}\right|\right) \\
& \geq \mathscr{G}:=\inf _{\substack{(j, k),(n, m) \in I^{N} \backslash A \\
(j, k) \neq(n, m)}}\left|\lambda_{j}^{u_{0}}-\lambda_{k}^{u_{0}}-\lambda_{n}^{u_{0}}+\lambda_{m}^{u_{0}}\right|>0
\end{aligned}
$$

where $A$ runs over the finite subsets of $I^{N}$ (we refer to the second point of the proof for further details on $\mathscr{G}^{\prime}$ ). The solvability of the moment problem (3.11) is guaranteed from Corollary A.9 for $T>\frac{2 \pi}{\mathscr{G}^{\prime}}$ by considering the sequence of numbers obtained by reordering

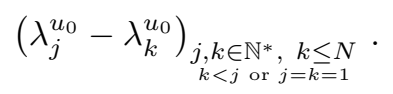

Indeed, $x_{1,1}^{u_{0}}=0$ and Remark B.9 ensures that $\lambda_{j}^{u_{0}}-\lambda_{k}^{u_{0}} \neq \lambda_{l}^{u_{0}}-\lambda_{m}^{u_{0}}$ for every $(j, k),(l, m) \in I^{N}$ (see (1.4)) such that $(j, k) \neq(n, m)$. In conclusion, the solvability of the moment problem implies the surjectivity of $\gamma^{u_{0}}$ and the inverse mapping theorem ensures the surjectivity of $\alpha^{u_{0}}$ in $Q_{\epsilon}^{N}$ for $T>0$ large and suitable $\epsilon$. The proof is achieved as discussed in Remark 3.2.

2) We show that the controllability ensured in 1) is valid for every positive time $T>0$ by proving that $\mathscr{G}^{\prime}=+\infty$. Let

$$
A^{M}:=\left\{(j, n) \in\left(\mathbb{N}^{*}\right)^{2} \mid j, n \geq M ; j \neq n\right\}, \quad M \in \mathbb{N}^{*} .
$$

Thanks to the identity (B.2) from the proof of Lemma B.4, for $\left|u_{0}\right|$ small enough and for $j>n$, we have

$$
\begin{aligned}
& \lambda_{N}^{u_{0}} \leq \lambda_{N}+O\left(\left|u_{0}\right|\right), \\
& \lambda_{j}^{u_{0}}-\lambda_{n}^{u_{0}} \geq \lambda_{j}-\lambda_{n}-O\left(\left|u_{0}\right|\right) \geq \pi^{2}(2 n+1)-O\left(\left|u_{0}\right|\right) .
\end{aligned}
$$

Hence, for every $K \in \mathbb{R}$, there exists $M_{K}>0$ large enough such that

$$
\inf _{(j, n) \in A^{M_{K}}}\left|\lambda_{j}^{u_{0}}-\lambda_{n}^{u_{0}}\right|>K
$$

Now,

$$
\begin{aligned}
\mathscr{G}^{\prime} & \geq \sup _{A \subset I^{N}}\left(\inf _{\substack{(j, k),(n, m) \in I^{N} \backslash A \\
(j, k) \neq(n, m)}}\left|\lambda_{j}^{u_{0}}-\lambda_{n}^{u_{0}}\right|-\left|\lambda_{k}^{u_{0}}+\lambda_{m}^{u_{0}}\right|\right) \\
& \geq \sup _{M \in \mathbb{N}^{*}}\left(\inf _{(j, n) \in A^{M}}\left|\lambda_{j}^{u_{0}}-\lambda_{n}^{u_{0}}\right|-2 \lambda_{N}^{u_{0}}\right)>0
\end{aligned}
$$

where $A$ are the subsets of $I^{N}$ defining $\mathscr{G}^{\prime}$. In conclusion, for $\left|u_{0}\right|$ small enough, the relations (3.12)-(3.13) yield

$$
\begin{aligned}
\mathscr{G}^{\prime} & \geq \lim _{M \rightarrow \infty}\left(\inf _{(j, n) \in A^{M}}\left|\lambda_{j}-\lambda_{n}\right|-2 \lambda_{N}-O\left(\left|u_{0}\right|\right)\right) \\
& \geq \lim _{M \rightarrow \infty} 2 M+1-2 N^{2} \pi^{2}-O\left(\left|u_{0}\right|\right)=+\infty .
\end{aligned}
$$

Finally, $\mathscr{G}^{\prime}=+\infty$ and then the local exact controllability proved in the first point of the proof holds for every positive time since the result is valid for every $T>\frac{2 \pi}{\mathscr{G}^{\prime}}$. 


\section{Simultaneous global exact controllability in projection}

4.1. Preliminaries. The common approach adopted in order to prove global exact controllability results consists in gathering the global approximate controllability and the local exact controllability. Nevertheless, this strategy can not be used to prove the controllability in projection as the propagator $\Gamma_{T}^{u}$ does not preserve the space $\pi_{N}(\Psi) H_{(0)}^{3}$ for any $\Psi:=\left(\psi_{j}\right)_{j \in \mathbb{N}^{*}} \subset H_{(0)}^{3}$. For instance, let $\Psi=\left(\psi_{j}\right)_{j \in \mathbb{N}^{*}}$ be an orthonormal system and $\psi^{1}, \psi^{2} \in H_{(0)}^{3}$ be unitarily equivalent. Even though there exist $T_{1}, T_{2}>0, u_{1} \in L^{2}\left(\left(0, T_{1}\right), \mathbb{R}\right)$ and $u_{2} \in L^{2}\left(\left(0, T_{1}\right), \mathbb{R}\right)$ such that

$$
\pi_{N}(\Psi) \Gamma_{T_{1}}^{u_{1}} \psi_{1}=\pi_{N}(\Psi) \Gamma_{T_{2}}^{u_{2}} \psi_{2}
$$

it is not guaranteed the existence $T>0$ and a control $u \in L^{2}((0, T), \mathbb{R})$ such that

$$
\pi_{N}(\Psi) \Gamma_{T}^{u} \psi_{1}=\pi_{N}(\Psi) \psi_{2} .
$$

To this purpose, we adopt an alternative strategy based on the result presented in the following subsection. There, we prove that the controllability in projection of infinite bilinear Schrödinger equations is equivalent to the controllability (without projecting) of a finite number of them. Hence, we ensure the simultaneous global exact controllability for $N \in \mathbb{N}^{*}(\mathrm{BSE})$ in $\left(H_{(0)}^{3}\right)^{N}$. In such space, we can concatenate and reverse dynamics as it is preserved by the dynamics. The result leads to Theorem 1.1.

\subsection{Equivalence between controllability of finite bilinear Schrödinger} equations and infinitely many equations in projection.

Proposition 4.1. The two following assertions are equivalent with $N \in \mathbb{N}^{*}$. (1) Let $\left(\psi_{j}^{1}\right)_{j \in \mathbb{N}^{*}}$ and $\left(\psi_{j}^{2}\right)_{j \in \mathbb{N}^{*}} \subset H_{(0)}^{3}$ be a couple of complete orthonormal systems of $\mathscr{H}$. Let $\widehat{\Gamma}$ be the unitary operator such that $\left(\widehat{\Gamma} \psi_{j}^{2}\right)_{j \in \mathbb{N}^{*}}=\left(\psi_{j}^{1}\right)_{j \in \mathbb{N}^{*}}$. For any $\Psi:=\left(\psi_{j}\right)_{j \leq N} \subset H_{(0)}^{3}$ orthonormal system of $\mathscr{H}$ such that $\left(\widehat{\Gamma} \psi_{j}\right)_{j \leq N} \subset H_{(0)}^{3}$, there exist $T>0$ and $u \in L^{2}((0, T), \mathbb{R})$ such that

$$
\left\langle\psi_{k}, \Gamma_{T}^{u} \psi_{j}^{1}\right\rangle_{L^{2}}=\left\langle\psi_{k}, \psi_{j}^{2}\right\rangle_{L^{2}}, \quad \forall j, k \in \mathbb{N}^{*}, k \leq N .
$$

In other words, the following identities are satisfied (with $\pi_{N}(\Psi)$ defined in (1.1)):

$$
\pi_{N}(\Psi) \Gamma_{T}^{u} \psi_{j}^{1}=\pi_{N}(\Psi) \psi_{j}^{2}, \quad \forall j \in \mathbb{N}^{*} .
$$

(2) Let $\left(\psi_{j}^{1}\right)_{j \leq N}$ and $\left(\psi_{j}^{2}\right)_{j \leq N} \subset H_{(0)}^{3}$ be a couple of orthonormal systems in $\mathscr{H}$. There exist $T>0$ and $u \in L^{2}((0, T), \mathbb{R})$ such that

$$
\Gamma_{T}^{u} \psi_{j}^{1}=\psi_{j}^{2}, \quad \forall j \leq N .
$$

Proof. (2) $\Longrightarrow(1)$ Let $\Psi^{3}:=\left(\psi_{j}^{3}\right)_{j \in \mathbb{N}^{*}} \in H_{(0)}^{3}$ be an orthonormal system. We consider $\left(\psi_{j}^{1}\right)_{j \in \mathbb{N}^{*}},\left(\psi_{j}^{2}\right)_{j \in \mathbb{N}^{*}} \subset H_{(0)}^{3}$ complete orthonormal systems. Let $\widehat{\Gamma} \in$ $U(\mathscr{H})$ be such that $\widehat{\Gamma} \psi_{j}^{2}=\psi_{j}^{1}$ and $\widehat{\Gamma} \psi_{k}^{3} \in H_{(0)}^{3}$ for every $k \leq N$. We notice that the controllability stated in the point (2) of Theorem 4.1 is also valid for the reversed dynamics discussed in Section 2.3. Hence, there exist $T>0$ and $u \in L^{2}((0, T), \mathbb{R})$ such that

$$
\widetilde{\Gamma}_{T}^{u} \psi_{k}^{3}=\widehat{\Gamma} \psi_{k}^{3}, \quad \forall k \leq N
$$

Thus,

$$
\left\langle\widetilde{\Gamma}_{T}^{u} \psi_{k}^{3}, \psi_{j}^{1}\right\rangle_{L^{2}}=\left\langle\widehat{\Gamma} \psi_{k}^{3}, \psi_{j}^{1}\right\rangle_{L^{2}}, \quad \forall j, k \in \mathbb{N}^{*}, k \leq N
$$


Let $\widetilde{u}$ be introduced in Section 2.3. The claim is proved since, for every $j, k \in \mathbb{N}^{*}$ with $k \leq N$,

$$
\left\langle\Gamma_{T}^{\widetilde{u}} \psi_{j}^{1}, \psi_{k}^{3}\right\rangle_{L^{2}}=\left\langle\psi_{j}^{1}, \widetilde{\Gamma}_{T}^{\widetilde{u}} \psi_{k}^{3}\right\rangle_{L^{2}}=\left\langle\psi_{j}^{1}, \widehat{\Gamma} \psi_{k}^{3}\right\rangle_{L^{2}}=\left\langle\psi_{j}^{2}, \psi_{k}^{3}\right\rangle_{L^{2}} .
$$

(1) $\Longrightarrow$ (2) Let $\left(\psi_{j}^{1}\right)_{j \leq N},\left(\psi_{j}^{2}\right)_{j \leq N} \subset H_{(0)}^{3}$ be two orthonormal systems of $\mathscr{H}$. We complete them by defining $\left(\psi_{j}^{1}\right)_{j \in \mathbb{N}^{*}},\left(\psi_{j}^{2}\right)_{j \in \mathbb{N}^{*}} \subset H_{(0)}^{3}$ two complete orthonormal systems of $\mathscr{H}$. Now, thanks to the point (1), there exist $T>0$ and $u \in$ $L^{2}((0, T), \mathbb{R})$ such that

$$
\pi_{N}\left(\Psi^{2}\right) \Gamma_{T}^{u} \psi_{j}^{1}=\pi_{N}\left(\Psi^{2}\right) \psi_{j}^{2}, \quad \forall j \in \mathbb{N}^{*} .
$$

As $\Psi^{2}$ is composed by orthogonal elements and $\Gamma_{T}^{u}$ is unitary, the claim is proved since

$$
\begin{cases}\Gamma_{T}^{u} \psi_{j}^{1}=\psi_{j}^{2}, & \forall j \leq N, \\ \pi_{N}\left(\Psi^{2}\right) \Gamma_{T}^{u} \psi_{j}^{1}=0, & \forall j>N .\end{cases}
$$

REMARK 4.2. The previous proof contains the reason why we need to impose a $H_{(0)}^{3}$-compatibility condition such as (1.5) in order to obtain the controllabilty in projection of infinitely many (BSE). In particular, let $T>0, u \in L^{2}((0, T), \mathbb{R})$, $\widehat{\Gamma} \in U(\mathscr{H}),\left(\psi_{j}\right)_{j \in \mathbb{N}^{*}} \subset H_{(0)}^{3}$ and $\left(\psi_{j}^{1}\right)_{j \in \mathbb{N}^{*}} \subset H_{(0)}^{3}$ be a complete orthonormal system of $\mathscr{H}$. We know that if for every $j, k \in \mathbb{N}^{*}$ and $k \leq N$, we have

$$
\left\langle\Gamma_{T}^{u} \psi_{j}^{1}, \psi_{k}\right\rangle_{L^{2}}=\left\langle\widehat{\Gamma} \psi_{j}^{1}, \psi_{k}\right\rangle_{L^{2}}, \quad \Longrightarrow \quad\left\langle\psi_{j}^{1}, \widetilde{\Gamma}_{T}^{\widetilde{u}} \psi_{k}\right\rangle_{L^{2}}=\left\langle\psi_{j}^{1}, \widehat{\Gamma} \psi_{k}\right\rangle_{L^{2}}
$$

The last relation is equivalent to $\widetilde{\Gamma}_{T}^{\widetilde{u}} \psi_{k}=\widehat{\Gamma} \psi_{k}$ for every $k \leq N$. Now, $\widetilde{\Gamma}_{T}^{\widetilde{u}}$ is the propagator of the reversed dynamics introduced in the previous section and it preserves $H_{(0)}^{3}$. This fact tells that the controllability in projection can be ensured only when a $H_{(0)}^{3}$-compatibility condition such as (1.5) is guaranteed. Namely, when $\widehat{\Gamma}$ and $\left(\psi_{j}\right)_{j \in \mathbb{N}^{*}}$ are such that $\widehat{\Gamma} \psi_{k} \in H_{(0)}^{3}$ for every $k \leq N$.

4.3. Simultaneous approximate controllability. In this section, we prove the simultaneous global approximate controllability for finite number of (BSE).

Definition 4.3. The problems (BSE) are said to be simultaneously globally approximately controllable in $H_{(0)}^{3}$ when, for every $N \in \mathbb{N}^{*}, \psi_{1}, \ldots, \psi_{N} \in H_{(0)}^{3}$, $\widehat{\Gamma} \in U(\mathscr{H})$ such that $\widehat{\Gamma} \psi_{1}, \ldots, \widehat{\Gamma} \psi_{N} \in H_{(0)}^{3}$ and $\epsilon>0$, there exist $T>0$ and $u \in L^{2}((0, T), \mathbb{R})$ such that $\left\|\Gamma_{T}^{u} \psi_{k}-\widehat{\Gamma} \psi_{k}\right\|_{(3)}<\epsilon$ for every $1 \leq k \leq N$.

TheOREM 4.4. Let B satisfy Assumptions I. The problems (BSE) are simultaneously globally approximately controllable in $H_{(0)}^{3}$.

Proof. In the point $\mathbf{1})$ of the proof, we suppose that $(A, B)$ admits a nondegenerate chain of connectedness (see [8, Definition 3]). We treat the general case in the point 2) of the proof. 
1) Preliminaries. Let $\pi_{m}$ be the orthogonal projector $\pi_{m}: \mathscr{H} \rightarrow \mathscr{H}_{m}:=$ $\operatorname{span}\left\{\phi_{j}: j \leq m\right\}$ for every $m \in \mathbb{N}^{*}$. Up to reordering of $\left(\phi_{k}\right)_{k \in \mathbb{N}^{*}}$, the couples $\left(\pi_{m} A \pi_{m}, \pi_{m} B \pi_{m}\right)$ for $m \in \mathbb{N}^{*}$ admit non-degenerate chains of connectedness in $\mathscr{H}_{m}$. Let $\|\cdot\|_{B V(T)}=\|\cdot\|_{B V((0, T), \mathbb{R})}$ and $\|\cdot\|\left\|_{(s)}:=\right\|\|\cdot\|_{L\left(H_{(0)}^{s}, H_{(0)}^{s}\right)}$ for $s>0$. Thanks to the validity of Assumptions I, we have $B: H_{(0)}^{2} \rightarrow H_{(0)}^{2}$. Let us denote

$$
S U\left(\mathscr{H}_{m}\right)=\left\{\Gamma \in U\left(\mathscr{H}_{m}\right):\left(\left\langle\phi_{j}, \Gamma \phi_{k}\right\rangle_{L^{2}}\right)_{j, k \leq m} \in S U(m)\right\} .
$$

Claim. For every $\epsilon>0$, there exist $N_{1} \in \mathbb{N}^{*}$ with $N_{1} \geq N$ and $\widetilde{\Gamma}_{N_{1}} \in U(\mathscr{H})$ such that $\pi_{N_{1}}(\Phi) \widetilde{\Gamma}_{N_{1}} \pi_{N_{1}}(\Phi) \in S U\left(\mathscr{H}_{N_{1}}\right)$ and

$$
\left\|\widetilde{\Gamma}_{N_{1}} \phi_{j}-\widehat{\Gamma} \phi_{j}\right\|_{L^{2}}<\epsilon, \quad \forall j \leq N .
$$

Let $N_{1} \in \mathbb{N}^{*}$ be such that $N_{1} \geq N$. We apply the orthonormalizing GramSchmidt process to $\left(\pi_{N_{1}}(\Phi) \widehat{\Gamma} \phi_{j}\right)_{j \leq N}$ and we define the sequence $\left(\widetilde{\phi}_{j}\right)_{j \leq N}$ that we complete in $\left(\widetilde{\phi}_{j}\right)_{j \leq N_{1}}$, an orthonormal basis of $\mathscr{H}_{N_{1}}$. We complete again such sequence in an orthonormal basis of $\mathscr{H}$ that we call $\left(\widetilde{\phi}_{j}\right)_{j \in \mathbb{N}^{*}}$. The operator $\widetilde{\Gamma}_{N_{1}}$ is the unitary map such that $\widetilde{\Gamma}_{N_{1}} \phi_{j}=\widetilde{\phi}_{j}$, for every $j \in \mathbb{N}^{*}$. In conclusion, we consider $N_{1}$ large enough such that the statement is verified.

Finite dimensional controllability. Let $T_{a d}$ be the set of $(j, k) \in\left\{1, \ldots, N_{1}\right\}^{2}$ such that $B_{j, k}:=\left\langle\phi_{j}, B \phi_{k}\right\rangle_{L^{2}} \neq 0$ and $\left|\lambda_{j}-\lambda_{k}\right|=\left|\lambda_{m}-\lambda_{l}\right|$ with $m, l \in \mathbb{N}^{*}$ implies $\{j, k\}=\{m, l\}$ for $B_{m, l}=0$. For every $(j, k) \in\left\{1, \ldots, N_{1}\right\}^{2}$ and $\theta \in[0,2 \pi)$, we define $E_{j, k}^{\theta}$ the $N_{1} \times N_{1}$ matrix with elements

$$
\left(E_{j, k}^{\theta}\right)_{j, k}=e^{i \theta}, \quad\left(E_{j, k}^{\theta}\right)_{k, j}=-e^{-i \theta}, \quad\left(E_{j, k}^{\theta}\right)_{l, m}=0,
$$

for $(l, m) \in\left\{1, \ldots, N_{1}\right\}^{2} \backslash\{(j, k),(k, j)\}$. Let

$$
E_{a d}=\left\{E_{j, k}^{\theta}:(j, k) \in T_{a d}, \theta \in[0,2 \pi)\right\}
$$

and $\operatorname{Lie}\left(E_{a d}\right)$. Fixed $v$ a piecewise constant control taking value in $E_{a d}$ and $\tau>0$, we introduce the control system on $S U\left(N_{1}\right)$

$$
\left\{\begin{array}{l}
\dot{x}(t)=x(t) v(t), \quad t \in(0, \tau), \\
x(0)=I d_{S U\left(N_{1}\right)} .
\end{array}\right.
$$

Claim. (4.2) is controllable, i.e. for $R \in S U\left(N_{1}\right)$, there exist $p \in \mathbb{N}^{*}, M_{1}, \ldots, M_{p} \in$ $E_{a d}, \alpha_{1}, \ldots, \alpha_{p} \in \mathbb{R}^{+}$such that $R=e^{\alpha_{1} M_{1}} \circ \ldots \circ e^{\alpha_{p} M_{p}}$.

For every $(j, k) \in\left\{1, \ldots, N_{1}\right\}^{2}$, we define the $N_{1} \times N_{1}$ matrices $R_{j, k}, C_{j, k}$ and $D_{j}$ as follows. For $(l, m) \in\left\{1, \ldots, N_{1}\right\}^{2} \backslash\{(j, k),(k, j)\}$, we have

$$
\begin{gathered}
\left(R_{j, k}\right)_{l, m}=0, \quad\left(R_{j, k}\right)_{j, k}=-\left(R_{j, k}\right)_{k, j}=1, \\
\left(C_{j, k}\right)_{l, m}=0, \quad\left(C_{j, k}\right)_{j, k}=\left(C_{j, k}\right)_{k, j}=i .
\end{gathered}
$$

Moreover, for $(l, m) \in\left\{1, \ldots, N_{1}\right\}^{2} \backslash\{(1,1),(j, j)\}$,

$$
\left(D_{j}\right)_{l, m}=0, \quad\left(D_{j}\right)_{1,1}=-\left(D_{j}\right)_{j, j}=i .
$$

We denote by $s u\left(N_{1}\right)$ the Lie algebra of $S U\left(N_{1}\right)$ and we consider its basis

$$
\mathbf{e}:=\left\{R_{j, k}\right\}_{j, k \leq N_{1}} \cup\left\{C_{j, k}\right\}_{j, k \leq N_{1}} \cup\left\{D_{j}\right\}_{j \leq N_{1}} .
$$

Thanks to [18, Theorem 6.1], the controllability of (4.2) is equivalent to prove that $\operatorname{Lie}\left(E_{a d}\right) \supseteq s u\left(N_{1}\right)$. The claim is valid as it is possible to obtain the matrices $R_{j, k}$, $C_{j, k}$ and $D_{j}$ for every $j, k \leq N_{1}$ by iterated Lie brackets of elements in $E_{a d}$. 
Finite dimensional estimates. The previous claim and the fact that the matrix $\left(\left\langle\phi_{j}, \widetilde{\Gamma}_{N_{1}} \phi_{k}\right\rangle_{L^{2}}\right)_{j, k \leq N_{1}} \in S U\left(N_{1}\right)$ ensure the existence of $p \in \mathbb{N}^{*}, M_{1}, \ldots, M_{p} \in E_{a d}$ and $\alpha_{1}, \ldots, \alpha_{p} \in \mathbb{R}^{+}$such that

$$
\left(\left\langle\phi_{j}, \widetilde{\Gamma}_{N_{1}} \phi_{k}\right\rangle_{L^{2}}\right)_{j, k \leq N_{1}}=e^{\alpha_{1} M_{1}} \circ \ldots \circ e^{\alpha_{p} M_{p}} .
$$

For every $l \leq p$, we call $\widehat{\Gamma}_{l}$ the operator in $S U\left(\mathscr{H}_{N_{1}}\right)$ such that $\left(\left\langle\phi_{j}, \widehat{\Gamma}_{l} \phi_{k}\right\rangle\right)_{j, k \leq N_{1}}=$ $e^{\alpha_{l} M_{l}}$. The identity (4.3) yields

$$
\pi_{N_{1}}(\Phi) \widetilde{\Gamma}_{N_{1}} \pi_{N_{1}}(\Phi)=\widehat{\Gamma}_{1} \circ \ldots \circ \widehat{\Gamma}_{p}
$$

Claim. For every $l \leq p$ and $\widehat{\Gamma}_{l}$ from (4.4), there exist $\left(T_{n}^{l}\right)_{l \in \mathbb{N}^{*}} \subset \mathbb{R}^{+}$and $\left(u_{n}^{l}\right)_{n \in \mathbb{N}^{*}}$ such that $u_{n}^{l}:\left(0, T_{n}^{l}\right) \rightarrow \mathbb{R}$ for every $n \in \mathbb{N}^{*}$ and

$$
\begin{gathered}
\lim _{n \rightarrow \infty}\left\|\Gamma_{T_{n}^{l}}^{u_{n}^{l}} \phi_{k}-\widehat{\Gamma}_{l} \phi_{k}\right\|_{L^{2}}=0, \quad \forall k \leq N_{1}, \\
\sup _{n \in \mathbb{N}^{*}}\left\|u_{n}^{l}\right\|_{B V\left(T_{n}\right)}<\infty, \quad \sup _{n \in \mathbb{N}^{*}}\left\|u_{n}^{l}\right\|_{L^{\infty}\left(\left(0, T_{n}\right), \mathbb{R}\right)}<\infty, \\
\sup _{n \in \mathbb{N}^{*}} T_{n}\left\|u_{n}^{l}\right\|_{L^{\infty}\left(\left(0, T_{n}\right), \mathbb{R}\right)}<\infty .
\end{gathered}
$$

We consider the results developed in [9, Section 3.1 \& Section 3.2] by Chambrion and leading to $[\mathbf{9}$, Proposition 6$]$ since $(A, B)$ admits a non-degenerate chain of connectedness (defined in [8, Definition 3]). Each $\widehat{\Gamma}_{l}$ corresponds to a rotation in a two dimensional space for every $l \in\{1, \ldots, p\}$. This work allows to explicit $\left(T_{n}^{l}\right)_{l \in \mathbb{N}^{*}} \subset \mathbb{R}^{+}$and $\left(u_{n}^{l}\right)_{n \in \mathbb{N}^{*}}$ satisfying (4.6) such that each $u_{n}^{l}:\left(0, T_{n}^{l}\right) \rightarrow \mathbb{R}$ and

$$
\lim _{n \rightarrow \infty}\left\|\pi_{N_{1}}(\Phi) \Gamma_{T_{n}^{l}}^{u_{n}^{l}} \phi_{k}-\widehat{\Gamma}_{l} \phi_{k}\right\|_{L^{2}}=0, \quad \forall k \leq N_{1} .
$$

As $\widehat{\Gamma}_{l} \in S U\left(\mathscr{H}_{N_{1}}\right)$, we have $\lim _{n \rightarrow \infty}\left\|\Gamma_{T_{n}^{l}}^{u_{n}^{l}} \phi_{k}-\widehat{\Gamma}_{l} \phi_{k}\right\|_{L^{2}}=0$ for $k \leq N_{1}$.

\section{Infinite dimensional estimates.}

Claim. There exist $K_{1}, K_{2}, K_{3}>0$ such that for every $\epsilon>0$, there exist $T>0$ and $u \in L^{2}((0, T), \mathbb{R})$ such that $\left\|\Gamma_{T}^{u} \phi_{k}-\widehat{\Gamma} \phi_{k}\right\|_{L^{2}}<\epsilon$ for every $k \leq N_{1}$ and

$$
\|u\|_{B V(T)} \leq K_{1}, \quad\|u\|_{L^{\infty}((0, T), \mathbb{R})} \leq K_{2}, \quad T\|u\|_{L^{\infty}((0, T), \mathbb{R})} \leq K_{3} .
$$

Let us assume that 1) (c) be valid with $p=2$. Nevertheless, the following result is valid for any $p \in \mathbb{N}^{*}$. By definition of $\widehat{\Gamma}_{1} \in S U\left(\mathscr{H}_{N_{1}}\right)$, for every $k \leq N_{1}$, there exist $l_{k} \leq N_{1}$ and $\alpha_{l_{k}} \in \mathbb{C}$ with $\left|\alpha_{l_{k}}\right|=1$ such that $\widehat{\Gamma}_{1} \phi_{k}=\alpha_{l_{k}} \phi_{l_{k}}$. Thanks to (4.5), for $n \in \mathbb{N}^{*}$ sufficiently large,

$$
\begin{aligned}
\left\|\Gamma_{T_{n}^{2}}^{u_{n}^{2}} \Gamma_{T_{n}^{1}}^{u_{n}^{1}} \phi_{k}-\widehat{\Gamma}_{2} \widehat{\Gamma}_{1} \phi_{k}\right\|_{L^{2}} \leq & \left\|\Gamma_{T_{n}^{2}}^{u_{n}^{2}}\right\|\left\|\Gamma_{T_{n}^{1}}^{u_{n}^{1}} \phi_{k}-\widehat{\Gamma}_{1} \phi_{k}\right\|_{L^{2}} \\
& +\left\|\alpha_{l_{k}} \Gamma_{T_{n}^{2}}^{u_{n}^{2}} \phi_{l_{k}}-\alpha_{l_{k}} \widehat{\Gamma}_{2} \phi_{l_{k}}\right\|_{L^{2}}<\epsilon, \quad \forall k \leq N_{1} .
\end{aligned}
$$

The identity (4.4) leads to the existence of $K_{1}, K_{2}, K_{3}>0$ such that for every $\epsilon>0$, there exist $T>0$ and $u \in L^{2}((0, T), \mathbb{R})$ such that $\left\|\Gamma_{T}^{u} \phi_{k}-\widetilde{\Gamma}_{N_{1}} \phi_{k}\right\|_{L^{2}}<\epsilon$ for every $k \leq N_{1}$ and

$$
\|u\|_{B V(T)} \leq K_{1}, \quad\|u\|_{L^{\infty}((0, T), \mathbb{R})} \leq K_{2}, \quad T\|u\|_{L^{\infty}((0, T), \mathbb{R})} \leq K_{3} .
$$

The relation (4.1) and the triangular inequality achieve the claim. 
Global approximate controllability with respect to the $L^{2}$-norm. Let us recall that $\left(\psi_{j}\right)_{j \leq N} \subset H_{(0)}^{3}$ and $\widehat{\Gamma} \in U(\mathscr{H})$ satisfies $\left(\widehat{\Gamma} \psi_{j}\right)_{j \leq N} \subset H_{(0)}^{3}$.

Claim. There exist $K_{1}, K_{2}, K_{3}>0$ such that for every $\epsilon>0$, there exist $T>0$ and $u \in L^{2}((0, T), \mathbb{R})$ such that $\left\|\Gamma_{T}^{u} \psi_{k}-\widehat{\Gamma} \psi_{k}\right\|_{L^{2}}<\epsilon$ for every $k \leq N$ and

$$
\|u\|_{B V(T)} \leq K_{1}, \quad\|u\|_{L^{\infty}((0, T), \mathbb{R})} \leq K_{2}, \quad T\|u\|_{L^{\infty}((0, T), \mathbb{R})} \leq K_{3} .
$$

We assume that $\left\|\psi_{j}\right\|_{L^{2}}=1$ for every $j \in \mathbb{N}^{*}$, but the same proof is also valid for the generic case. From the previous claim, there exist two controls respectively steering $\left(\phi_{j}\right)_{j \leq N}$ close to $\left(\psi_{j}\right)_{j \leq N}$ and $\left(\phi_{j}\right)_{j \leq N}$ close to $\left(\widehat{\Gamma} \psi_{j}\right)_{j \leq N}$ thanks to the fact that $N_{1} \geq N$. Vice versa, thanks to the time reversibility (see Section 2.3), there exists a control steering $\left(\psi_{j}\right)_{j \leq N}$ close to $\left(\phi_{j}\right)_{j \leq N}$. In other words, there exist $T_{1}, T_{2}>0, u_{1} \in L^{2}\left(\left(0, T_{1}\right), \mathbb{R}\right)$ and $u_{2} \in L^{2}\left(\left(0, T_{2}\right), \mathbb{R}\right)$ such that

$$
\left\|\Gamma_{T_{1}}^{u_{1}} \psi_{j}-\phi_{j}\right\|_{L^{2}}<\frac{\epsilon}{2}, \quad\left\|\Gamma_{T_{2}}^{u_{2}} \phi_{j}-\widehat{\Gamma} \psi_{j}\right\|_{L^{2}}<\frac{\epsilon}{2}, \quad \forall j \leq N .
$$

The chosen controls $u_{1}$ and $u_{2}$ satisfy (4.7). The claim is proven as

$\left\|\Gamma_{T_{2}}^{u_{2}} \Gamma_{T_{1}}^{u_{1}} \psi_{j}-\widehat{\Gamma} \psi_{j}\right\|_{L^{2}} \leq\left\|\Gamma_{T_{2}}^{u_{2}} \Gamma_{T_{1}}^{u_{1}} \psi_{j}-\Gamma_{T_{2}}^{u_{2}} \phi_{j}\right\|_{L^{2}}+\left\|\Gamma_{T_{2}}^{u_{2}} \phi_{j}-\widehat{\Gamma} \psi_{j}\right\|_{L^{2}}<\epsilon, \quad \forall j \leq N$.

Global approximate controllability with respect to the $H_{(0)}^{3}$-norm.

Claim. There exist $T>0$ and $u \in L^{2}((0, T), \mathbb{R})$ such that $\left\|\Gamma_{T}^{u} \psi_{k}-\widehat{\Gamma} \psi_{k}\right\|_{(3)}<\epsilon$ for every $k \leq N$.

We consider the propagation of regularity developed by Kato in [11]. We notice that $i(A+u(t) B-i c)$ is maximal dissipative in $H_{(0)}^{2}$ for suitable $c>0$. Let $\lambda>c$ and $\widehat{H}_{(0)}^{4}:=D(A(i \lambda-A)) \equiv H_{(0)}^{4}$. We know that $B: \widehat{H}_{(0)}^{4} \subset H_{(0)}^{2} \rightarrow H_{(0)}^{2}$ and the arguments of Remark 2.1 imply that $B \in L\left(\widehat{H}_{(0)}^{4}, H_{(0)}^{2}\right)$. For $T>0$ and $u \in B V((0, T), \mathbb{R})$, we have $\left\|u(t) B(i \lambda-A)^{-1}\right\|_{(2)}<1$ and

$$
\begin{aligned}
M & :=\sup _{t \in[0, T]}\left\|(i \lambda-A-u(t) B)^{-1}\right\|_{L\left(H_{(0)}^{2}, \widehat{H}_{(0)}^{4}\right)} \\
& \leq \sup _{t \in[0, T]} \sum_{l=1}^{+\infty}\left\|\left(u(t) B(i \lambda-A)^{-1}\right)^{l}\right\|_{(2)}<+\infty .
\end{aligned}
$$

We know $\|k+f(\cdot)\|_{B V((0, T), \mathbb{R})}=\|f\|_{B V((0, T), \mathbb{R})}$ for $f \in B V((0, T), \mathbb{R})$ and $k \in \mathbb{R}$. Equivalently,

$$
\begin{aligned}
N & \left.:=\|i \lambda-A-u(\cdot) B\|_{B V\left([0, T], L\left(\widehat{H}_{(0)}^{4}, H_{(0)}^{2}\right)\right.}\right) \\
& =\|u\|_{B V(T)}\|B\|_{L\left(\widehat{H}_{(0)}^{4}, H_{(0)}^{2}\right)}<+\infty .
\end{aligned}
$$

We call $U_{t}^{u}$ the propagator generated by $A+u B-i c$ such that $U_{t}^{u} \psi=e^{-c t} \Gamma_{t}^{u} \psi$. Thanks to [11, Section 3.10], for every $\psi \in H_{(0)}^{4}$, it follows

$$
\left\|(A+u(T) B-i \lambda) U_{t}^{u} \psi\right\|_{(2)} \leq M e^{M N}\|(A-i \lambda) \psi\|_{(2)}
$$

which implies, for $C_{1}:=\left\|A(A+u(T) B-i \lambda)^{-1}\right\|_{(2)}<\infty$,

$$
\left\|\Gamma_{T}^{u} \psi\right\|_{(4)} \leq C_{1} M e^{M N+c T}\|\psi\|_{(4)} .
$$


For every $T>0, u \in B V((0, T), \mathbb{R})$ and $\psi \in H_{(0)}^{4}$, there exists $C(K)>0$ depending on $K=\left(\|u\|_{B V(T)},\|u\|_{L^{\infty}((0, T), \mathbb{R})}, T\|u\|_{L^{\infty}((0, T), \mathbb{R})}\right)$ such that $\left\|\Gamma_{T}^{u} \psi\right\|_{(4)} \leq$ $C(K)\|\psi\|_{(4)}$. When (4.6) is verified, there exists $C>0$ such that, for every $n \in \mathbb{N}^{*}$,

$$
\left\|\Gamma_{T_{n}^{l}}^{u_{n}^{l}}\right\|_{(4)} \leq C .
$$

For every $\psi \in H_{(0)}^{4}$, from the Cauchy-Schwarz inequality, we have $\|A \psi\|_{L^{2}}^{2} \leq$ $\left\langle A^{2} \psi, \psi\right\rangle_{L^{2}} \leq\left\|A^{2} \psi\right\|_{L^{2}}\|\psi\|_{L^{2}}$ and $\left\|A^{\frac{3}{2}} \psi\right\|_{L^{2}}^{4} \leq\left(\left\langle A^{2} \psi, A \psi\right\rangle_{L^{2}}\right)^{2} \leq\left\|A^{2} \psi\right\|_{L^{2}}^{2}\|A \psi\|_{L^{2}}^{2}$, which imply

$$
\|\psi\|_{(3)}^{8} \leq\|\psi\|_{L^{2}}^{2}\|\psi\|_{(4)}^{6} .
$$

In conclusion, the claim of the global approximate controllability with respect to the $L^{2}$-norm and the relations (4.8)-(4.9) ensure the claim.

2) Conclusion. Assume that $(A, B)$ does not admit a non-degenerate chain of connectedness. We decompose

$$
A+u(\cdot) B=\left(A+u_{0} B\right)+u_{1}(\cdot) B, \quad u_{0} \in \mathbb{R}, \quad u_{1} \in L^{2}((0, T), \mathbb{R}) .
$$

We notice that, if $(A, B)$ satisfies Assumptions I, then Remark B.7 and Remark B.9 are valid. We consider $u_{0}$ belonging to the neighborhoods provided by such remarks and we denote by $\left(\phi_{k}^{u_{0}}\right)_{k \in \mathbb{N}^{*}}$ a complete orthonormal system of $\mathscr{H}$ made by eigenfunctions of $A+u_{0} B$. Thanks to the first point of Remark B.9, the couple $\left(A+u_{0} B, B\right)$ admits a non-degenerate chain of connectedness. The step $\left.\mathbf{1}\right)$ of the proof can be repeated by considering the sequence $\left(\phi_{k}^{u_{0}}\right)_{k \in \mathbb{N}^{*}}$ instead of $\left(\phi_{k}\right)_{k \in \mathbb{N}^{*}}$ and the spaces $D\left(\left|A+u_{0} B\right|^{\frac{3}{2}}\right)$ in substitution of $H_{(0)}^{3}$. The claim is equivalently proved since, thanks to Remark B.7, there exist $C_{1}, C_{2}>0$ such that

$$
C_{1}\left\|\left|A+u_{0} B\right|^{\frac{3}{2}} \psi\right\| \leq\|\psi\|_{(3)} \leq C_{2}\left\|\left|A+u_{0} B\right|^{\frac{3}{2}} \psi\right\|, \quad \forall \psi \in H_{(0)}^{3} .
$$

4.4. Proof of Theorem 1.1. In the current subsection, we provide the proof of Theorem 1.1 which requires the following proposition.

Proposition 4.5. Let $N \in \mathbb{N}^{*}$ and $B$ satisfy Assumptions I. For any $\left(\psi_{k}^{1}\right)_{k \leq N}$, $\left(\psi_{k}^{2}\right)_{k \leq N} \subset H_{(0)}^{3}$ orthonormal systems, there exist $T>0, u \in L^{2}((0, T), \mathbb{R})$ and $\left(\theta_{k}\right)_{k \leq N} \subset \mathbb{R}$ such that

$$
\Gamma_{T}^{u} \psi_{k}^{1}=e^{i \theta_{k}} \psi_{k}^{2}, \quad \forall k \leq N
$$

Proof. Let $N \in \mathbb{N}^{*}$ and let $u_{0} \in \mathbb{R}$ belong to the neighborhoods provided by Lemma B.5, Lemma B.6 and Remark B.9. Let $\widetilde{\alpha}^{u_{0}}$ be the map with elements

$$
\begin{cases}\frac{\overline{\widehat{\alpha}_{j, j}\left(u_{1}\right)}}{\left|\widehat{\alpha}_{j, j}\left(u_{1}\right)\right|} \widehat{\alpha}_{k, j}\left(u_{1}\right), & \forall j, k \in \mathbb{N}^{*}, j, k \leq N, \\ \widehat{\alpha}_{k, j}\left(u_{1}\right), & \forall j, k \in \mathbb{N}^{*}, k>N, j \leq N .\end{cases}
$$

The proof of Proposition 3.3 can be repeated in order to prove the local surjectivity of $\widetilde{\alpha}^{u_{0}}$ for every $T>0$, instead of $\alpha^{u_{0}}$ introduced in (3.7). The discussion from Remark 3.2 implies that this result corresponds to the simultaneous local exact controllability up to phases of $N$ problems (BSE) in the neighborhood

$$
O_{\epsilon, T}^{N}:=\left\{\left(\psi_{j}\right)_{j \leq N} \subset H_{(0)}^{3} \mid\left\langle\psi_{j}, \psi_{k}\right\rangle_{L^{2}}=\delta_{j, k} ; \sup _{j \leq N}\left\|\psi_{j}-\phi_{j}^{u_{0}}\right\|_{(3)}^{2}<\epsilon\right\}
$$


with $\epsilon>0$. Hence, for any $\left(\psi_{k}\right)_{k \leq N} \in O_{\epsilon, T}^{N}$, there exist $u \in L^{2}((0, T), \mathbb{R})$ and $\left(\theta_{j}\right)_{j \leq N} \subset \mathbb{R}$ such that

$$
\Gamma_{T}^{u} \phi_{j}^{u_{0}}=e^{i \theta_{j}} \psi_{j}, \quad \forall j \leq N
$$

Thanks to Theorem 4.4, we have the following result. For any $\left(\psi_{j}^{1}\right)_{j \leq N} \subset H_{(0)}^{3}$ composed by orthonormal elements, there exist $T_{1}>0$ and $u_{1} \in L^{2}\left(\left(0, T_{1}\right), \mathbb{R}\right)$ such that, for all $j \leq N$,

$$
\left\|\Gamma_{T_{1}}^{u_{1}} \psi_{j}^{1}-\phi_{j}^{u_{0}}\right\|_{(3)}<\frac{\epsilon}{N} \quad \Longrightarrow \quad\left(\Gamma_{T_{1}}^{u_{1}} \psi_{j}^{1}\right)_{j \leq N} \in O_{\epsilon, T}^{N} .
$$

The local controllability is also valid for the reversed dynamics (see Section 2.3) and for every $T>0$, there exist $u \in L^{2}((0, T), \mathbb{R})$ and $\left(\theta_{j}\right)_{j \leq N} \subset \mathbb{R}$ such that

$$
\left(\Gamma_{T_{1}}^{u_{1}} \psi_{j}^{1}\right)_{j \leq N}=\left(e^{i \theta_{j}} \widetilde{\Gamma}_{T}^{u} \phi_{j}^{u_{0}}\right)_{j \leq N} \quad \Longrightarrow \quad\left(e^{-i \theta_{j}} \Gamma_{T}^{\widetilde{u}} \Gamma_{T_{1}}^{u_{1}} \psi_{j}^{1}\right)_{j \leq N}=\left(\phi_{j}^{u_{0}}\right)_{j \leq N} .
$$

Then, there exist $T_{2}>0$ and $u_{2} \in L^{2}\left(\left(0, T_{2}\right), \mathbb{R}\right)$ such that $\left(e^{-i \theta_{j}} \Gamma_{T_{2}}^{u_{2}} \psi_{j}^{1}\right)_{j \leq N}=$ $\left(\phi_{j}^{u_{0}}\right)_{j \leq N}$. Now, the same property is valid for the reversed dynamics of (2.4) and, for every $\left(\psi_{j}^{2}\right)_{j \leq N} \subset H_{(0)}^{3}$ composed by orthonormal elements, there exist $T_{3}>0$, $u_{3} \in L^{2}\left(\left(0, T_{3}\right), \mathbb{R}\right)$ and $\left(\theta_{j}^{\prime}\right)_{j \leq N} \subset \mathbb{R}$ such that $\left(e^{-i \theta_{j}^{\prime}} \widetilde{\Gamma}_{T_{3}}^{u_{3}} \psi_{j}^{2}\right)_{j \leq N}=\left(\phi_{j}^{u_{0}}\right)_{j \leq N}$. In conclusion, for $\widetilde{u}_{3}(\cdot)=u_{3}\left(T_{3}-\cdot\right)$, the proof is achieved as

$$
\left(e^{-i\left(\theta_{j}-\theta_{j}^{\prime}\right)} \Gamma_{T_{3}}^{\widetilde{u}_{3}} \Gamma_{T_{2}}^{u_{2}} \psi_{j}^{1}\right)_{j \leq N}=\left(\psi_{j}^{2}\right)_{j \leq N} .
$$

Proof of Theorem 1.1. The claim is proved as the implication $(\mathbf{2}) \Longrightarrow(\mathbf{1})$ in the proof of Theorem 4.1 thanks to the validity of Proposition 4.5.

\section{Global exact controllability in projection for density matrices}

Let $\psi^{1}, \psi^{2} \in \mathscr{H}$. We define the rank one operator $\left|\psi^{1}\right\rangle\left\langle\psi^{2}\right|$ such that $\left|\psi^{1}\right\rangle\left\langle\psi^{2}\right| \psi=$ $\psi^{1}\left\langle\psi^{2}, \psi\right\rangle_{L^{2}}$ for every $\psi \in \mathscr{H}$. For any $\widehat{\Gamma} \in U(\mathscr{H})$, we have

$$
\widehat{\Gamma}\left|\psi^{1}\right\rangle\left\langle\psi^{2}|=| \widehat{\Gamma} \psi^{1}\right\rangle\left\langle\psi^{2}|, \quad| \psi^{1}\right\rangle\left\langle\psi^{2}\left|\widehat{\Gamma}^{*}=\right| \psi^{1}\right\rangle\left\langle\widehat{\Gamma} \psi^{2}\right| .
$$

Let $\mathscr{H}$ be an infinite dimensional Hilbert space. In quantum mechanics, any statistical ensemble can be described by a wave function (pure state) or by a density matrix (mixed state) which is a positive operator of trace 1 . For any density matrix $\rho$, there exists a sequence $\left(\psi_{j}\right)_{j \in \mathbb{N}^{*}} \subset \mathscr{H}$ such that

$$
\rho=\sum_{j \in \mathbb{N}^{*}} l_{j}\left|\psi_{j}\right\rangle\left\langle\psi_{j}\right|, \quad \sum_{j \in \mathbb{N}^{*}} l_{j}=1, \quad l_{j} \geq 0, \quad \forall j \in \mathbb{N}^{*} .
$$

The sequence $\left(\psi_{j}\right)_{j \in \mathbb{N}^{*}}$ is a set of eigenvectors of $\rho$ and $\left(l_{j}\right)_{j \in \mathbb{N}^{*}}$ are the corresponding eigenvalues. If there exists $j_{0} \in \mathbb{N}^{*}$ such that $l_{j_{0}}=1$ and $l_{j}=0$ for each $j \neq j_{0}$, then the corresponding density matrix represents a pure state up to a phase. For this reason, the density matrices formalism is said to be an extension of the common formulation of the quantum mechanics in terms of wave function. We also notice that for any density matrix $\rho$ and a complete orthonormal system $\left(\psi_{j}\right)_{j \in \mathbb{N}^{*}}$ in $\mathscr{H}$, there exists a positive hermitian matrix $\left(\rho_{j, k}\right)_{j, k \in \mathbb{N}^{*}}$ such that

$$
\rho=\sum_{j, k \in \mathbb{N}^{*}} \rho_{j, k}\left|\psi_{j}\right\rangle\left\langle\psi_{k}\right| .
$$


Now, for any other density matrix $\widetilde{\rho}$, there exists an orthonormal system $\left(\widetilde{\psi}_{j}\right)_{j \in \mathbb{N}^{*}}$, such that

$$
\widetilde{\rho}=\sum_{j, k \in \mathbb{N}^{*}} \rho_{j, k}\left|\widetilde{\psi}_{j}\right\rangle\left\langle\widetilde{\psi}_{k}\right| .
$$

Let us consider $T>0$ and a time dependent self-adjoint operator $H(t)$ (called Hamiltonian) for $t \in(0, T)$. The dynamics of a general density matrix $\rho$ is described by the Von Neumann equation

$$
\left\{\begin{array}{lr}
i \frac{d \rho}{d t}(t)=[H(t), \rho(t)], & t \in(0, T), \\
\rho(0)=\rho^{0}, & ([H, \rho]=H \rho-\rho H),
\end{array}\right.
$$

for $\rho^{0}$ the initial solution of the problem. The solution is $\rho(t)=U_{t} \rho(0) U_{t}^{*}$, where $U_{t}$ is the unitary propagator generated by $H(t)$. In the present work, we have $\mathscr{H}=L^{2}((0,1), \mathbb{C}), H(t)=A+u(t) B$ and $U_{t}$ corresponds to $\Gamma_{t}^{u}$. In this framework, the problem (5.4) is said to be globally exactly controllable if, for any couple of density matrices $\rho^{1}$ and $\rho^{2}$, there exist $T>0$ and $u \in L^{2}((0, T), \mathbb{R})$ such that

$$
\rho^{2}=\Gamma_{T}^{u} \rho^{1}\left(\Gamma_{T}^{u}\right)^{*} .
$$

Thanks to the decomposition (5.1), the controllability of (5.4) is equivalent (up to phases) to the simultaneous controllability of the infinite bilinear Schrödinger equations (BSE). This idea is behind the following theorem which follows from Corollary 1.2.

Theorem 5.1. Let B satisfy Assumptions I. Let $\rho^{1}$ and $\rho^{2}$ be two density matrices with eigenfunctions in $H_{(0)}^{3}$ and $\widehat{\Gamma} \in U(\mathscr{H})$ be such that

$$
\rho^{1}=\widehat{\Gamma} \rho^{2} \widehat{\Gamma}^{*} .
$$

1) Let $\Psi:=\left(\psi_{j}\right)_{j \in \mathbb{N}^{*}}$ be an orthonormal system composed by the eigenfunctions of $\rho^{2}$. For any $N \in \mathbb{N}^{*}$, there exist $T>0$ and $u \in L^{2}((0, T), \mathbb{R})$ such that

$$
\pi_{N}(\Psi) \Gamma_{T}^{u} \rho^{1}\left(\Gamma_{T}^{u}\right)^{*} \pi_{N}(\Psi)=\pi_{N}(\Psi) \rho^{2} \pi_{N}(\Psi) .
$$

2) Let $\Psi:=\left(\psi_{j}\right)_{j \leq N} \subset H_{(0)}^{3}$ be an orthonormal system such that $\left(\widehat{\Gamma} \psi_{j}\right)_{j \leq N} \subset H_{(0)}^{3}$ with $N \in \mathbb{N}^{*}$. Let $\left(\rho_{j, k}\right)_{j, k \leq N}$ be the positive hermitian matrix such that

$$
\pi_{N}(\Psi) \rho^{2} \pi_{N}(\Psi)=\sum_{j, k \leq N} \rho_{j, k}\left|\psi_{j}\right\rangle\left\langle\psi_{k}\right| .
$$

There exist $T>0, u \in L^{2}((0, T), \mathbb{R})$ and $\left(\theta_{j, k}\right)_{j, k \leq N}$ such that

$$
\pi_{N}(\Psi) \Gamma_{T}^{u} \rho^{1}\left(\Gamma_{T}^{u}\right)^{*} \pi_{N}(\Psi)=\sum_{j, k \leq N} e^{i \theta_{j, k}} \rho_{j, k}\left|\psi_{j}\right\rangle\left\langle\psi_{k}\right| .
$$

Proof. 1) Let $\left(\psi_{j}^{1}\right)_{j \in \mathbb{N}^{*}} \subset H_{(0)}^{3}$ be an orthonormal system made by eigenfunctions of $\rho^{1}$. We have

$$
\rho^{1}=\sum_{j=1}^{\infty} l_{j}\left|\psi_{j}^{1}\right\rangle\left\langle\psi_{j}^{1}\left|, \quad \rho^{2}=\sum_{j=1}^{\infty} l_{j}\right| \psi_{j}\right\rangle\left\langle\psi_{j}\right| .
$$

The sequence $\left(l_{j}\right)_{j \in \mathbb{N}^{*}} \subset \mathbb{R}^{+}$corresponds to the spectrum of $\rho^{1}$ and $\rho^{2}$. Now, thanks to Corollary 1.2 , there exist $T>0, u \in L^{2}((0, T), \mathbb{R})$ and $\left(\theta_{j}\right)_{j \leq N}$ such that 
$\pi_{N}(\Psi) \Gamma_{T}^{u} \psi_{j}^{1}=e^{i \theta_{j}} \pi_{N}(\Psi) \psi_{j}$ for every $j \leq N$, while $\pi_{N}(\Psi) \Gamma_{T}^{u} \psi_{j}^{1}=\pi_{N}(\Psi) \psi_{j}$ for every $j>N$. Thus,

$$
\begin{aligned}
\pi_{N}(\Psi) \Gamma_{T}^{u} \rho^{1}\left(\Gamma_{T}^{u}\right)^{*} \pi_{N}(\Psi) & =\sum_{j=1}^{N} l_{j}\left|e^{i \theta_{j}} \pi_{N}(\Psi) \Gamma_{T}^{u} \psi_{j}^{1}\right\rangle\left\langle\psi_{j}^{1} \Gamma_{T}^{u} \pi_{N}(\Psi) e^{i \theta_{j}}\right| \\
& +\sum_{j=N+1}^{\infty} l_{j}\left|\pi_{N}(\Psi) \Gamma_{T}^{u} \psi_{j}^{1}\right\rangle\left\langle\psi_{j}^{1} \Gamma_{T}^{u} \pi_{N}(\Psi)\right| \\
& =\sum_{j=1}^{\infty} l_{j} \pi_{N}(\Psi)\left|\psi_{j}\right\rangle\left\langle\psi_{j}\right| \pi_{N}(\Psi)=\pi_{N}(\Psi) \rho^{2} \pi_{N}(\Psi) .
\end{aligned}
$$

2) The second point of the theorem follows from the same arguments of the first one. In particular, the statement follows by decomposing $\rho^{2}$ with respect to $\left(\psi_{j}\right)_{j \in \mathbb{N}^{*}}$ as done in (5.2). Such step provides a positive hermitian matrix $\left(\rho_{j, k}\right)_{j, k \in \mathbb{N}^{*}}$. Now, we define $\left(\psi_{j}^{1}\right)_{j \in \mathbb{N}^{*}}$ as the orthonormal system such that (5.3) is valid for the density

matrix $\rho^{1}$. The claim is proved by simultaneously steering $\left(\psi_{j}^{1}\right)_{j \in \mathbb{N}^{*}}$ in $\left(\psi_{j}\right)_{j \in \mathbb{N}^{*}}$ with respect to the projector $\pi(\Psi)$ by using Corollary 1.2 .

\section{Conclusion}

In this manuscript, we study the controllability of the infinite bilinear Schrödinger equations (BSE) at the same time $T$, with one unique control $u$ and by projecting onto suitable finite dimensional subspaces of $\mathscr{H}$. The first result of the work is the simultaneous local exact controllability of infinite bilinear Schrodinger equations in projection in any positive $T>0$. The property is stated by Theorem 3.1 and Proposition 3.3. Our second achievement is Theorem 4.1 which shows that the simultaneous global exact controllability of the (BSE) in projection onto a suitable $N$ dimensional space is equivalent to the controllability of $N$ problems (BSE) (without projecting). Finally, we prove Theorem 1.1 which states the simultaneous global exact controllability in projection of infinite (BSE). The result is guaranteed when the orthogonal projector is defined by an orthonormal systems verifying a $H_{(0)}^{3}$-compatibility condition exposed in (1.5). In conclusion, we rephrase the main results in terms of density matrices.

Here, one could wonder if the techniques developed in this manuscript can be applied to study the controllability of infinite (BSE) (without projecting). Nevertheless, a direct application is not possible. Indeed, one of the crucial points of our strategy is the possibility of decoupling with a uniform gap the eigenvalues resonances appearing in the proof of Theorem 3.1 (see Section 3.2 for further details). We obtain such property via perturbation theory techniques thanks to the fact that eigenvalues resonances are finite when we project onto finite dimensional spaces.

In any case, a possible approach that might lead to the controllability of infinite (BSE) is the following. As already done in our work, one could perturb in order to decouple the eigenvalues resonances appearing in the proof of the simultaneous local exact controllability. In such framework, we do not expect to have a uniform spectral gap and then the Haraux's Theorem A.8 can not be applied. As a consequence, the solvability of the moment problem (such as (3.11)) appearing in this proof can not be achieved in $\ell^{2}$. Nevertheless, we do not exclude the possibility of proving its solvability in some spaces $h^{s}$ with $s \in[0,1)$ (defined in 2.1) by using more refined techniques as the Beurling's Theorem [13, Theorem 9.2] (see also $[\mathbf{1}$, 
Chapter I.2]). If such result would be valid, then the well-posedness of the (BSE) can be provided in $H_{(0)}^{3+s}$ by imposing slightly more regularity on the operator $B$ and we might conclude the proof as done in the current work.

\section{Appendix A. Moment problem}

We denote by $\langle\cdot, \cdot\rangle_{L^{2}(0, T)}$ the scalar product in $L^{2}((0, T), \mathbb{C})$ with $T>0$.

Definition A.1. Let $\left(f_{k}\right)_{k \in \mathbb{Z}}$ be a family of functions in $L^{2}((0, T), \mathbb{C})$ with $T>$ 0 . The family $\left(f_{k}\right)_{k \in \mathbb{Z}}$ is said to be minimal if and only if $f_{k} \notin \overline{\operatorname{span}\left\{f_{j}: j \neq k\right\}} L^{2}$ for every $k \in \mathbb{Z}$.

Definition A.2. A biorthogonal family to $\left(f_{k}\right)_{k \in \mathbb{Z}} \subset L^{2}((0, T), \mathbb{C})$ is a sequence of functions $\left(g_{k}\right)_{k \in \mathbb{Z}}$ in $L^{2}((0, T), \mathbb{C})$ such that $\left\langle f_{k}, g_{j}\right\rangle_{L^{2}(0, T)}=\delta_{k, j}$ for every $k, j \in \mathbb{Z}$.

REMARK A.3. When $\left(f_{k}\right)_{k \in \mathbb{Z}}$ is minimal, there exists an unique biorthogonal family $\left(g_{k}\right)_{k \in \mathbb{Z}}$ to $\left(f_{k}\right)_{k \in \mathbb{Z}}$ belonging to $X:=\overline{\operatorname{span}\left\{f_{j}: j \in \mathbb{Z}\right\}}{ }^{2}$. Its existence follows from the fact that $\left(g_{k}\right)_{k \in \mathbb{Z}}$ can be constructed by setting

$$
g_{k}=\left(f_{k}-\tilde{\pi}_{k} f_{k}\right)\left\|f_{k}-\pi_{k} f_{k}\right\|_{L^{2}(0, T)}^{-2}, \quad \forall k \in \mathbb{Z}
$$

where $\tilde{\pi}_{k}$ is the orthogonal projector onto $\overline{\operatorname{span}\left\{f_{j}: j \neq k\right\}}{ }^{2}$. The unicity follows as, for any biorthogonal family $\left(g_{k}^{1}\right)_{k \in \mathbb{Z}}$ in $X$, we have $\left\langle g_{k}-g_{k}^{1}, f_{j}\right\rangle_{L^{2}(0, T)}=0$ for every $j, k \in \mathbb{Z}$, which implies $g_{k}=g_{k}^{1}$ for every $k \in \mathbb{Z}$.

REMARK A.4. If a sequence of functions $\left(f_{k}\right)_{k \in \mathbb{Z}} \subset L^{2}((0, T), \mathbb{C})$ admits a biorthogonal family $\left(g_{k}\right)_{k \in \mathbb{Z}}$, then it is minimal. Indeed, if we assume that there exists $k \in \mathbb{Z}$ such that $f_{k} \in \overline{\operatorname{span}\left\{f_{j}: j \neq k\right\}} L^{2}$, then the relations $\left\langle f_{k}, g_{j}\right\rangle_{L^{2}(0, T)}=0$ for every $j \in \mathbb{Z} \backslash\{k\}$ would imply $\left\langle f_{k}, g_{k}\right\rangle_{L^{2}(0, T)}=0$ which is absurd.

Definition A.5. Let $\left(f_{k}\right)_{k \in \mathbb{Z}}$ be a family of functions in $L^{2}((0, T), \mathbb{C})$ with $T>0$. The family $\left(f_{k}\right)_{k \in \mathbb{Z}}$ is a Riesz basis of $\overline{\operatorname{span}\left\{f_{j}: j \in \mathbb{Z}\right\}} L^{2}$ if and only if it is isomorphic to an orthonormal system.

Remark A.6. Let $\left(f_{k}\right)_{k \in \mathbb{Z}}$ be a Riesz basis of $X:=\overline{\operatorname{span}\left\{f_{j}: j \in \mathbb{Z}\right\}}{ }^{2}$. The sequence $\left(f_{k}\right)_{k \in \mathbb{Z}}$ is minimal and its biorthogonal family is uniquely defined in $X$ thanks to Remark A.3. Finally, this biorthogonal family forms a Riesz basis of $X$.

Now, we provide an important property on the Riesz basis proved in $[\mathbf{4}, \mathrm{Ap}$ pendix B.1].

Proposition A.7. [4, Appendix B; Proposition 19] Let $\left(f_{k}\right)_{k \in \mathbb{Z}}$ be a family of functions in $L^{2}((0, T), \mathbb{C})$ with $T>0$. The sequence $\left(f_{k}\right)_{k \in \mathbb{Z}}$ is a Riesz basis of $\overline{\operatorname{span}\left\{f_{k}: k \in \mathbb{Z}\right\}}{ }^{L^{2}}$ if and only if there exist $C_{1}, C_{1}>0$ such that

$$
C_{1}\|\mathbf{x}\|_{\ell^{2}}^{2} \leq \int_{0}^{T}\left|\sum_{k \in \mathbb{Z}} x_{k} f_{k}\right|^{2} d s \leq C_{2}\|\mathbf{x}\|_{\ell^{2}}^{2}, \quad \forall \mathbf{x}:=\left(x_{k}\right)_{k \in \mathbb{Z}} \in \ell^{2}(\mathbb{Z}, \mathbb{C}) .
$$

We are finally ready to present the so-called Haraux's Theorem. 
Theorem A.8. [13, Theorem 4.6] Let $\left(\omega_{k}\right)_{k \in \mathbb{Z}}$ be a family of real numbers satisfying the uniform gap condition $\mathscr{G}:=\inf _{k \neq j}\left|\omega_{k}-\omega_{j}\right|>0$. Let

$$
\mathscr{G}^{\prime}:=\sup _{K \subset \mathbb{Z}} \inf _{\substack{j \in \mathbb{Z} \backslash K \\ k \neq j}}\left|\omega_{k}-\omega_{j}\right|>0,
$$

where $K$ runs over the finite subsets of $\mathbb{Z}$. For every bounded interval $|I|>\frac{2 \pi}{G^{\prime}}$, there exist $C_{1}, C_{2}>0$ such that

$$
C_{1} \sum_{k \in \mathbb{Z}}\left|x_{k}\right|^{2} \leq \int_{I}|u(t)|^{2} d t \leq C_{2} \sum_{k \in \mathbb{Z}}\left|x_{k}\right|^{2},
$$

for every $u(t)=\sum_{k \in \mathbb{Z}} x_{k} e^{i \omega_{k} t}$ with $\left(x_{k}\right)_{k \in \mathbb{Z}} \in \ell^{2}(\mathbb{Z}, \mathbb{C})$.

The following corollary follows from the Haraux's Theorem and provides the solvability of suitable moment problems as (3.2) and (3.11).

Corollary A.9. Let $\left(\lambda_{k}\right)_{k \in \mathbb{N}^{*}}$ be an ordered sequence of real numbers such that $\lambda_{1}=0$ and $\mathscr{G}:=\inf _{k \neq j}\left|\lambda_{k}-\lambda_{j}\right|>0$. Let

$$
\mathscr{G}^{\prime}:=\sup _{K \subset \mathbb{N}^{*}} \inf _{\substack{k \in \mathbb{N}^{*} \backslash K \\ k \neq j}}\left|\lambda_{k}-\lambda_{j}\right|,
$$

where $K$ runs over the finite subsets of $\mathbb{N}^{*}$. Fixed $T>2 \pi / \mathscr{G}^{\prime}$, for every $\left(x_{k}\right)_{k \in \mathbb{N}^{*}} \in$ $\ell^{2}(\mathbb{C})$, there exists $u \in L^{2}((0, T), \mathbb{R})$ such that

$$
x_{k}=\int_{0}^{T} u(s) e^{-i \lambda_{k} s} d s, \quad \forall k \in \mathbb{N}^{*} .
$$

Proof. For $k \in \mathbb{N}^{*}$, we call $\omega_{k}=\lambda_{k}$, while we impose $\omega_{k}=-\lambda_{-k}$ for $-k \in$ $\mathbb{N}^{*} \backslash\{1\}$. We call $\mathbb{Z}^{*}=\mathbb{Z} \backslash\{0\}$. The sequence $\left(\omega_{k}\right)_{k \in \mathbb{Z}^{*} \backslash\{-1\}}$ satisfies the hypotheses of Theorem A.8 for

$$
\sup _{K \subset \mathbb{Z}^{*} \backslash\{-1\}} \inf _{\substack{k, j \in\left(\mathbb{Z}^{*} \backslash\{-1\}\right) \backslash K \\ k \neq j}}\left|\omega_{k}-\omega_{j}\right|=\mathscr{G}^{\prime},
$$

where $K$ runs over the finite subsets of $\mathbb{Z}^{*} \backslash\{-1\}$. Proposition A.7 and Theorem A.8 ensure that the sequence $\left(e^{i \omega_{k} t}\right)_{k \in \mathbb{Z}^{*} \backslash\{-1\}}$ is a Riesz basis of

$$
X:=\overline{\operatorname{span}\left\{e^{i \omega_{k} t}: k \in \mathbb{Z}^{*} \backslash\{-1\}\right\}}{ }^{2} .
$$

Thanks to Remark A.6, its unique biorthogonal family $\left(v_{k}\right)_{k \in \mathbb{Z}^{*} \backslash\{-1\}}$ in $X$ is also a Riesz basis of $X$. Thanks to Proposition A.7, there exist $C_{1}, C_{2}>0$ such that

$$
C_{1} \sum_{k \in \mathbb{Z}^{*} \backslash\{-1\}}\left|x_{k}\right|^{2} \leq\|u\|_{L^{2}(0, T)}^{2} \leq C_{2} \sum_{k \in \mathbb{Z}^{*} \backslash\{-1\}}\left|x_{k}\right|^{2},
$$

with $u(t)=\sum_{k \in \mathbb{Z}^{*} \backslash\{-1\}} x_{k} v_{k}(t)$ and $\left(x_{k}\right)_{k \in \mathbb{Z}^{*} \backslash\{-1\}} \in \ell^{2}\left(\mathbb{Z}^{*} \backslash\{-1\}, \mathbb{C}\right)$. Now,

$$
u=\sum_{k \in \mathbb{Z}^{*} \backslash\{-1\}} v_{k}\left\langle e^{i \omega_{k} t}, u\right\rangle_{L^{2}(0, T)}
$$

since $\left(e^{i \omega_{k} t}\right)_{k \in \mathbb{Z}^{*} \backslash\{-1\}}$ and $\left(v_{k}\right)_{k \in \mathbb{Z}^{*} \backslash\{-1\}}$ are reciprocally biorthogonal. Hence,

$$
C_{1} \sum_{k \in \mathbb{Z}^{*} \backslash\{-1\}}\left|\left\langle e^{i \omega_{k} t}, u\right\rangle_{L^{2}(0, T)}\right|^{2} \leq\|u\|_{L^{2}(0, T)}^{2} \leq C_{2} \sum_{k \in \mathbb{Z}^{*} \backslash\{-1\}}\left|\left\langle e^{i \omega_{k} t}, u\right\rangle_{L^{2}(0, T)}\right|^{2} .
$$

The last relation yields the invertibility of the map

$$
F: u \in X \longmapsto\left(\left\langle e^{i \omega_{k} t}, u\right\rangle_{L^{2}(0, T)}\right)_{k \in \mathbb{Z}^{*} \backslash\{-1\}} \in \ell^{2}\left(\mathbb{Z}^{*} \backslash\{-1\}, \mathbb{C}\right) .
$$


Fixed $\left(x_{k}\right)_{k \in \mathbb{N}^{*}} \in \ell^{2}(\mathbb{C})$. We call $\left(\widetilde{x}_{k}\right)_{k \in \mathbb{Z}^{*} \backslash\{-1\}} \in \ell^{2}\left(\mathbb{Z}^{*} \backslash\{-1\}, \mathbb{C}\right)$ the sequence such that $\widetilde{x}_{k}=x_{k}$ for $k \in \mathbb{N}^{*}$, while $\widetilde{x}_{k}=\bar{x}_{-k}$ for $-k \in \mathbb{N}^{*} \backslash\{1\}$. For $T>2 \pi / \mathscr{G}^{\prime}$, the invertibility of the map $F$ ensures the existence of $u \in L^{2}((0, T), \mathbb{C})$ such that

$$
\widetilde{x}_{k}=\int_{0}^{T} u(s) e^{-i \omega_{k} s} d s
$$

for every $k \in \mathbb{Z}^{*} \backslash\{-1\}$. Thus,

$$
\left\{\begin{array}{l}
x_{k}=\int_{0}^{T} u(s) e^{-i \lambda_{k} s} d s=\int_{0}^{T} \bar{u}(s) e^{-i \lambda_{k} s} d s, \quad \forall k \in \mathbb{N}^{*} \backslash\{1\}, \\
x_{1}=\int_{0}^{T} u(s) d s .
\end{array}\right.
$$

Finally, if $x_{1} \in \mathbb{R}$, then (A.1) is valid with respect to a function $u$ which is real.

\section{Appendix B. Analytic Perturbation}

Let us consider the problem (3.5) and the eigenvalues $\left(\lambda_{j}^{u_{0}}\right)_{j \in \mathbb{N}^{*}}$ of the operator $A+u_{0} B$. When $B$ is a bounded symmetric operator satisfying Assumptions I and $A=-\Delta$ is the Laplacian with Dirichlet type boundary conditions $D(A)=$ $H^{2}((0,1), \mathbb{C}) \cap H_{0}^{1}((0,1), \mathbb{C})$, thanks to $[\mathbf{1 2}$, Theorem VII.2.6] and [12, Theorem VII.3.9], the following proposition follows.

Proposition B.1. Let B satisfy Assumptions I. There exists a neighborhood $D(0)$ of $u=0$ in $\mathbb{R}$ small enough where the maps $u \mapsto \lambda_{j}^{u}$ are analytic for every $j \in \mathbb{N}^{*}$.

The next lemma proves the existence of perturbations, which do not shrink too much the eigenvalues gaps.

Lemma B.2. Let B satisfy Assumptions I. There exists a neighborhood D(0) in $\mathbb{R}$ of $u=0$ such that, for each $u_{0} \in D(0)$, there exists $r>0$ such that, for every $j \in \mathbb{N}^{*}$,

$$
\mu_{j}:=\frac{\lambda_{j}+\lambda_{j+1}}{2} \in \rho\left(A+u_{0} B\right), \quad\left\|\left(A+u_{0} B-\mu_{j}\right)^{-1}\right\| \leq r .
$$

Proof. Let $D(0)$ be the neighborhood provided by Proposition B.1. We know $\left(A-\mu_{j}\right)$ is invertible in a bounded operator and $\mu_{j} \in \rho(A)$ (resolvent set of $A$ ). Let $\delta:=\min _{j \in \mathbb{N}^{*}}\left|\lambda_{j+1}-\lambda_{j}\right|$. We know that $\left\|\left|\left(A-\mu_{j}\right)^{-1} \|\right| \leq \sup _{k \in \mathbb{N}^{*}} \frac{1}{\left|\mu_{j}-\lambda_{k}\right|}=\right.$ $\frac{2}{\left|\lambda_{j+1}-\lambda_{j}\right|} \leq \frac{2}{\delta}$. Thus, for $u_{0} \in D(0)$,

$$
\left\|\left(A-\mu_{j}\right)^{-1} u_{0} B\right\||\leq| u_{0}\left|\left\|\left|\left(A-\mu_{j}\right)^{-1}\left\|\left|\|B\| \leq \frac{2}{\delta}\right| u_{0} \mid\right\| B \|\right.\right.\right.
$$

and if $\left|u_{0}\right| \leq \frac{\delta(1-\epsilon)}{2\|B\|}$ for $\epsilon \in(0,1)$, then $\left\|\left(A-\mu_{j}\right)^{-1} u_{0} B\right\| \leq 1-\epsilon$. The operator $\left(A+u_{0} B-\mu_{j}\right)$ is invertible and $\left\|\left(A+u_{0} B-\mu_{j}\right)^{-1}\right\| \leq \frac{2}{\delta \epsilon}$ as $\left\|\left(A+u_{0} B-\mu_{j}\right) \psi\right\|_{L^{2}} \geq$ $\left\|\left(A-\mu_{j}\right) \psi\right\|_{L^{2}}-\left\|u_{0} B \psi\right\|_{L^{2}} \geq \frac{\delta}{2}\|\psi\|_{L^{2}}-\frac{\delta(1-\epsilon)}{2}\|\psi\|_{L^{2}}$ for every $\psi \in D(A)$. The parameter $r$ stated in the lemma corresponds to $2 /(\delta \epsilon)$, while the neighborhood is $\left\{u_{0} \in D(0):\left|u_{0}\right| \leq \delta(1-\epsilon) /(2\|B\| \mid)\right\}$.

Lemma B.3. Let B satisfy Assumptions $I$ and $P_{\phi_{k}}^{\perp}$ be the projector onto the orthogonal space of $\phi_{k}$. There exists a neighborhood $D(0)$ of 0 in $\mathbb{R}$ such that

$$
\left(A+u_{0} P_{\phi_{k}}^{\perp} B-\lambda_{k}^{u_{0}}\right)
$$

is invertible with bounded inverse from $D(A) \cap \phi_{k}^{\perp}$ to $\phi_{k}^{\perp}$ for every $u_{0} \in D(0)$ and $k \in \mathbb{N}^{*}$. 
Proof. Let $D(0)$ be the neighborhood provided by Lemma B.2. For any $u_{0} \in D(0)$, one can consider the decomposition $\left(A+u_{0} P_{\phi_{k}}^{\perp} B-\lambda_{k}^{u_{0}}\right)=\left(A-\lambda_{k}^{u_{0}}\right)+$ $u_{0} P_{\phi_{k}}^{\perp} B$. The operator $A-\lambda_{k}^{u_{0}}$ is invertible with bounded inverse when it acts on the orthogonal space of $\phi_{k}$ and we estimate ||$\left(\left.\left(A-\lambda_{k}^{u_{0}}\right)\right|_{\phi_{\frac{1}{L}}}\right)^{-1} u_{0} P_{\phi_{k}}^{\perp} B \| \mid$. However, for every $\psi \in D(A) \cap \operatorname{Ran}\left(P_{\phi_{k}}^{\perp}\right)$ such that $\|\psi\|_{L^{2}}=1$, we have

$$
\left\|\left(A-\lambda_{k}^{u_{0}}\right) \psi\right\|_{L^{2}} \geq \min \left\{\left|\lambda_{k+1}-\lambda_{k}^{u_{0}}\right|,\left|\lambda_{k}^{u_{0}}-\lambda_{k-1}\right|\right\}\|\psi\|_{L^{2}} .
$$

Let $\delta_{k}:=\min \left\{\left|\lambda_{k+1}-\lambda_{k}^{u_{0}}\right|,\left|\lambda_{k}^{u_{0}}-\lambda_{k-1}\right|\right\}$. Thanks to Lemma B.2, for $\left|u_{0}\right|$ small enough, $\lambda_{k}^{u_{0}} \in\left(\frac{\lambda_{k-1}+\lambda_{k}}{2}, \frac{\lambda_{k}+\lambda_{k+1}}{2}\right)$ and then

$$
\delta_{k} \geq \min \left\{\left|\lambda_{k+1}-\frac{\lambda_{k}+\lambda_{k+1}}{2}\right|,\left|\frac{\lambda_{k-1}+\lambda_{k}}{2}-\lambda_{k-1}\right|\right\} \geq \frac{(2 k-1) \pi^{2}}{2}>k .
$$

Afterwards,

$$
\left\|\left(\left.\left(A-\lambda_{k}^{u_{0}}\right)\right|_{\phi_{k}^{\perp}}\right)^{-1} u_{0} P_{\phi_{k}}^{\perp} B\right\| \leq \frac{1}{\delta_{k}}\left|u_{0}\right|\||B|\|
$$

and, if $\left|u_{0}\right| \leq(1-r) \frac{\delta_{k}}{\|\| B \|} \leq \frac{(1-r)}{\|B\|}$ for $r \in(0,1)$, then it follows

$$
\left\|\left(\left.\left(A-\lambda_{k}^{u_{0}}\right)\right|_{\phi_{k}^{\perp}}\right)^{-1} u_{0} P_{\phi_{k}}^{\perp} B\right\| \leq(1-r)<1 .
$$

The operator $A_{k}:=\left(A-\lambda_{k}^{u_{0}}+u_{0} P_{\phi_{k}}^{\perp} B\right)$ is invertible when it acts on the orthogonal space of $\phi_{k}$ and, for every $\psi \in D(A)$ and $r=\frac{1}{2}$,

$$
\begin{aligned}
\left\|A_{k} \psi\right\|_{L^{2}} & \geq\left\|\left(A-\lambda_{k}^{u_{0}}\right) \psi\right\|_{L^{2}}-\left\|u_{0} P_{\phi_{k}}^{\perp} B \psi\right\|_{L^{2}} \\
& \geq \delta_{k}\|\psi\|_{L^{2}}-\left\|u_{0} P_{\phi_{k}}^{\perp} B\right\|\|\psi\|_{L^{2}} \geq \frac{1}{2}\|\psi\|_{L^{2}} .
\end{aligned}
$$

In conclusion, $\left\|\mid\left(\left.\left(A-\lambda_{k}^{u_{0}}+u_{0} P_{\phi_{k}}^{\perp} B\right)\right|_{\phi_{k}^{\perp}}\right)^{-1}\right\| \leq 2$ for every $k \in \mathbb{N}^{*}$.

Lemma B.4. Let B satisfy Assumptions I. There exists a neighborhood D(0) of 0 in $\mathbb{R}$ such that, for any $u_{0} \in D(0)$, we have $\lambda_{j}^{u_{0}} \neq 0$ and there exist two constants $C_{1}, C_{2}>0$ such that

$$
C_{1} \lambda_{j} \leq \lambda_{j}^{u_{0}} \leq C_{2} \lambda_{j}, \quad \forall j \in \mathbb{N}^{*} .
$$

Proof. Let $u_{0} \in D(0)$ for $D(0)$ the neighborhood provided by Lemma B.3. We decompose the eigenfunction $\phi_{j}^{u_{0}}=a_{j} \phi_{j}+\eta_{j}$, where $a_{j}$ is an orthonormalizing constant and $\eta_{j}$ is orthogonal to $\phi_{j}$. Hence $\lambda_{k}^{u_{0}} \phi_{k}^{u_{0}}=\left(A+u_{0} B\right)\left(a_{k} \phi_{k}+\eta_{k}\right)$ and $\lambda_{k}^{u_{0}} a_{k} \phi_{k}+\lambda_{k}^{u_{0}} \eta_{k}=A a_{k} \phi_{k}+A \eta_{k}+u_{0} B a_{k} \phi_{k}+u_{0} B \eta_{k}$. By projecting onto the orthogonal space of $\phi_{k}$,

$$
\lambda_{k}^{u_{0}} \eta_{k}=A \eta_{k}+u_{0} P_{\phi_{k}}^{\perp} B a_{k} \phi_{k}+u_{0} P_{\phi_{k}}^{\perp} B \eta_{k} .
$$

However, Lemma B.3 ensures that $A+u_{0} P_{\phi_{k}}^{\perp} B-\lambda_{k}^{u_{0}}$ is invertible with bounded inverse when it acts on the orthogonal space of $\phi_{k}$ and then

$$
\eta_{k}=-a_{k}\left(\left.\left(A+u_{0} P_{\phi_{k}}^{\perp} B-\lambda_{k}^{u_{0}}\right)\right|_{\phi_{k}^{\perp}}\right)^{-1} u_{0} P_{\phi_{k}}^{\perp} B \phi_{k} .
$$

Now,

$$
\begin{aligned}
\lambda_{j}^{u_{0}} & =\left\langle a_{j} \phi_{j}+\eta_{j},\left(A+u_{0} B\right)\left(a_{j} \phi_{j}+\eta_{j}\right)\right\rangle_{L^{2}}=\left|a_{j}\right|^{2} \lambda_{j}+u_{0}\left\langle a_{j} \phi_{j}, B a_{j} \phi_{j}\right\rangle_{L^{2}} \\
& +\left\langle a_{j} \phi_{j},\left(A+u_{0} B\right) \eta_{j}\right\rangle_{L^{2}}+\left\langle\eta_{j},\left(A+u_{0} B\right) a_{j} \phi_{j}\right\rangle_{L^{2}}+\left\langle\eta_{j},\left(A+u_{0} B\right) \eta_{j}\right\rangle_{L^{2}} .
\end{aligned}
$$


By using the relation (B.1),

$$
\begin{aligned}
\left\langle\eta_{j},\left(A+u_{0} B\right) \eta_{j}\right\rangle_{L^{2}} & =\left\langle\eta_{j},\left(A+u_{0} P_{\phi_{k}}^{\perp} B-\lambda_{j}^{u_{0}}\right) \eta_{j}\right\rangle_{L^{2}}+\lambda_{j}^{u_{0}}\left\|\eta_{j}\right\|_{L^{2}}^{2} \\
& =\lambda_{j}^{u_{0}}\left\|\eta_{j}\right\|_{L^{2}}^{2}-a_{j}\left\langle\eta_{j}, u_{0} P_{\phi_{j}}^{\perp} B \phi_{j}\right\rangle_{L^{2}} .
\end{aligned}
$$

However, $\left\langle\phi_{j},\left(A+u_{0} B\right) \eta_{j}\right\rangle_{L^{2}}=u_{0}\left\langle\phi_{j}, B \eta_{j}\right\rangle_{L^{2}}=u_{0}\left\langle P_{\phi_{j}}^{\perp} B \phi_{j}, \eta_{j}\right\rangle_{L^{2}}$ and $\left\langle\eta_{j},(A+\right.$ $\left.\left.u_{0} B\right) \phi_{j}\right\rangle_{L^{2}}=u_{0}\left\langle\eta_{j}, P_{\phi_{j}}^{\perp} B \phi_{j}\right\rangle_{L^{2}}$. Thus, the last relations yields

$$
\lambda_{j}^{u_{0}}=\left|a_{j}\right|^{2} \lambda_{j}+u_{0}\left|a_{j}\right|^{2} B_{j, j}+\lambda_{j}^{u_{0}}\left\|\eta_{j}\right\|_{L^{2}}^{2}+u_{0} \overline{a_{j}}\left\langle P_{\phi_{j}}^{\perp} B \phi_{j}, \eta_{j}\right\rangle_{L^{2}} .
$$

One can notice that $\left|a_{j}\right| \in[0,1]$ and $\left\|\eta_{j}\right\|_{L^{2}}$ are uniformly bounded in $j$. We show that the first accumulates at 1 and the second at 0 . Indeed, from the proof of Lemma (B.3) and the relation (B.1), there exists $C_{1}>0$ such that

$$
\left\|\eta_{j}\right\|_{L^{2}}^{2} \leq\left|u_{0}\right|^{2}\left\|\left|\left(\left.\left(A+u_{0} P_{\phi_{j}}^{\perp} B-\lambda_{j}^{u_{0}}\right)\right|_{\phi_{j}^{\perp}}\right)^{-1}\left\|\left.\right|^{2}\left|a_{j}\right|^{2}\right\| B \phi_{j} \|_{L^{2}}^{2} \leq \frac{C_{1}}{j^{2}}\right.\right.
$$

for $r \in(0,1)$, which implies that $\lim _{j \rightarrow \infty}\left\|\eta_{j}\right\|_{L^{2}}=0$. Afterwards, by contradiction, if $\left|a_{j}\right|$ does not converge to 1 , then there exists $\left(a_{j_{k}}\right)_{k \in \mathbb{N}^{*}}$ a subsequence of $\left(a_{j}\right)_{j \in \mathbb{N}^{*}}$ such that $\left|a_{j_{\infty}}\right|:=\lim _{k \rightarrow \infty}\left|a_{j_{k}}\right| \in[0,1)$. Now, we have

$1=\lim _{k \rightarrow \infty}\left\|\phi_{j_{k}}^{u_{0}}\right\|_{L^{2}} \leq \lim _{k \rightarrow \infty}\left|a_{j_{k}}\right|\left\|\phi_{j_{k}}\right\|_{L^{2}}+\left\|\eta_{j_{k}}\right\|_{L^{2}}=\lim _{k \rightarrow \infty}\left|a_{j_{k}}\right|+\left\|\eta_{j_{k}}\right\|_{L^{2}}=\left|a_{j_{\infty}}\right|<1$

that is absurd. Then, $\lim _{j \rightarrow \infty}\left|a_{j}\right|=1$. From (B.2), it follows that there exist two constants $C_{1}, C_{2}>0$ such that, for each $j \in \mathbb{N}^{*}, C_{1} \lambda_{j} \leq \lambda_{j}^{u_{0}} \leq C_{2} \lambda_{j}$ for $\left|u_{0}\right|$ small enough. The relation also implies that $\lambda_{j}^{u_{0}} \neq 0$ for every $j \in \mathbb{N}^{*}$ and $\left|u_{0}\right|$ small enough.

Lemma B.5. Let $B$ satisfy Assumptions $I$. For every $N \in \mathbb{N}^{*}$, there exist a neighborhood $D(0)$ of 0 in $\mathbb{R}$ and $\widetilde{C}_{N}>0$ such that, for any $u_{0} \in D(0)$, we have

$$
\left|\left\langle\phi_{k}^{u_{0}}, B \phi_{j}^{u_{0}}\right\rangle_{L^{2}}\right| \geq \frac{\widetilde{C}_{N}}{k^{3}}, \quad \forall k, j \in \mathbb{N}^{*}, j \leq N .
$$

Proof. We start by choosing $k \in \mathbb{N}^{*}$ such that $k \neq j$ and $u_{0} \in D(0)$ for $D(0)$ the neighborhood provided by Lemma B.4. Thanks to Assumptions I, we have

$$
\begin{aligned}
& \left|\left\langle\phi_{k}^{u_{0}}, B \phi_{j}^{u_{0}}\right\rangle_{L^{2}}\right|=\left|\left\langle a_{k} \phi_{k}+\eta_{k}, B\left(a_{j} \phi_{j}+\eta_{j}\right)\right\rangle_{L^{2}}\right| \\
& \geq C_{N} \frac{\overline{a_{k}} a_{j}}{k^{3}}-\left|\overline{a_{k}}\left\langle\phi_{k}, B \eta_{j}\right\rangle_{L^{2}}+a_{j}\left\langle\eta_{k}, B \phi_{j}\right\rangle_{L^{2}}+\left\langle\eta_{k}, B \eta_{j}\right\rangle_{L^{2}}\right| .
\end{aligned}
$$

1) Expansion of $\left\langle\eta_{k}, B \phi_{j}\right\rangle_{L^{2}},\left\langle\phi_{k}, B \eta_{j}\right\rangle_{L^{2}}$ and $\left\langle\eta_{k}, B \eta_{j}\right\rangle_{L^{2}}$ : Thanks to (B.1),

$$
\left\langle\eta_{k}, B \phi_{j}\right\rangle_{L^{2}}=\left\langle-a_{k}\left(\left.\left(A+u_{0} P_{\phi_{k}}^{\perp} B-\lambda_{k}^{u_{0}}\right)\right|_{\phi_{k}^{\perp}}\right)^{-1} u_{0} P_{\phi_{k}}^{\perp} B \phi_{k}, P_{\phi_{k}}^{\perp} B \phi_{j}\right\rangle_{L^{2}}
$$

for every $k \in \mathbb{N}^{*}$ and $j \leq N$, while the operator $\left(\left.\left(A+u_{0} P_{\phi_{k}}^{\perp} B-\lambda_{k}^{u_{0}}\right)\right|_{\phi_{k}^{\perp}}\right)^{-1}$ corresponds to

$$
\left(\left(A-\lambda_{k}^{u_{0}}\right) P_{\phi_{k}}^{\perp}\right)^{-1} \sum_{n=0}^{\infty}\left(u_{0}\left(\left(A-\lambda_{k}^{u_{0}}\right) P_{\phi_{k}}^{\perp}\right)^{-1} P_{\phi_{k}}^{\perp} B P_{\phi_{k}}^{\perp}\right)^{n}
$$

for $\left|u_{0}\right|$ small enough. For

$$
M_{k}:=\sum_{n=0}^{\infty}\left(u_{0}\left(\left(A-\lambda_{k}^{u_{0}}\right) P_{\phi_{k}}^{\perp}\right)^{-1} P_{\phi_{k}}^{\perp} B\right)^{n} P_{\phi_{k}}^{\perp},
$$


we have

$$
\left\langle\eta_{k}, B \phi_{j}\right\rangle_{L^{2}}=-u_{0}\left\langle a_{k} M_{k} B \phi_{k},\left(\left(A-\lambda_{k}^{u_{0}}\right) P_{\phi_{k}}^{\perp}\right)^{-1} P_{\phi_{k}}^{\perp} B \phi_{j}\right\rangle_{L^{2}} .
$$

Thanks to $B: D(A) \rightarrow D(A)$, for every $k \in \mathbb{N}^{*}$ and $j \leq N$,

$$
\begin{aligned}
\left(\left(A-\lambda_{k}^{u_{0}}\right) P_{\phi_{k}}^{\perp}\right)^{-1} P_{\phi_{k}}^{\perp} B \phi_{j} & =P_{\phi_{k}}^{\perp} B\left(\left(A-\lambda_{k}^{u_{0}}\right) P_{\phi_{k}}^{\perp}\right)^{-1} \phi_{j} \\
& -\left[P_{\phi_{k}}^{\perp} B,\left(\left(A-\lambda_{k}^{u_{0}}\right) P_{\phi_{k}}^{\perp}\right)^{-1} P_{\phi_{k}}^{\perp}\right] \phi_{j} \\
& =P_{\phi_{k}}^{\perp} B\left(\left(A-\lambda_{k}^{u_{0}}\right) P_{\phi_{k}}^{\perp}\right)^{-1} \phi_{j} \\
& -\left(\left(A-\lambda_{k}^{u_{0}}\right) P_{\phi_{k}}^{\perp}\right)^{-1} P_{\phi_{k}}^{\perp}[B, A]\left(\left(A-\lambda_{k}^{u_{0}}\right) P_{\phi_{k}}^{\perp}\right)^{-1} \phi_{j} .
\end{aligned}
$$

For $\widetilde{B}_{k}:=\left(\left(A-\lambda_{k}^{u_{0}}\right) P_{\phi_{k}}^{\perp}\right)^{-1} P_{\phi_{k}}^{\perp}[B, A]$, we have

$$
\left(\left(A-\lambda_{k}^{u_{0}}\right) P_{\phi_{k}}^{\perp}\right)^{-1} P_{\phi_{k}}^{\perp} B \phi_{j}=P_{\phi_{k}}^{\perp}\left(B+\widetilde{B}_{k}\right)\left(\lambda_{j}-\lambda_{k}^{u_{0}}\right)^{-1} \phi_{j}
$$

and, for every $k \in \mathbb{N}^{*}$ and $j \leq N$,

$$
\left\langle\eta_{k}, B \phi_{j}\right\rangle_{L^{2}}=-\frac{u_{0}}{\lambda_{j}-\lambda_{k}^{u_{0}}}\left\langle a_{k} M_{k} B \phi_{k},\left(B+\widetilde{B}_{k}\right) \phi_{j}\right\rangle_{L^{2}}
$$

For every $k \in \mathbb{N}^{*}$ and $j \leq N$, we obtain

$$
\begin{aligned}
& \left|\left\langle\eta_{k}, B \eta_{j}\right\rangle_{L^{2}}\right|=\left|\left\langle B \eta_{k}, \eta_{j}\right\rangle_{L^{2}}\right|=\mid\left\langle u_{0} a_{k} B\left(\left(A-\lambda_{k}^{u_{0}}\right) P_{\phi_{k}}^{\perp}\right)^{-1} M_{k} B \phi_{k},\right. \\
& \left.u_{0} a_{j}\left(\left(A-\lambda_{j}^{u_{0}}\right) P_{\phi_{j}}^{\perp}\right)^{-1} M_{j} B \phi_{j}\right\rangle_{L^{2}}|=| \frac{a_{j} \overline{a_{k}} u_{0}^{2}}{\lambda_{k}-\lambda_{j}^{u_{0}}}\left\langle\phi_{k}, L_{k, j} \phi_{j}\right\rangle_{L^{2}} \mid
\end{aligned}
$$

with $L_{k, j}:=\left(A-\lambda_{j}^{u_{0}}\right) B M_{k}\left(\left(A-\lambda_{k}^{u_{0}}\right) P_{\phi_{k}}^{\perp}\right)^{-1} P_{\phi_{k}}^{\perp} B\left(\left(A-\lambda_{j}^{u_{0}}\right) P_{\phi_{j}}^{\perp}\right)^{-1} M_{j} B$. Now, there exists $\epsilon>0$ such that $\left|a_{l}\right| \in(\epsilon, 1)$ for every $l \in \mathbb{N}^{*}$. Thanks to (B.5), (B.6) and (B.4), there exists $\widehat{C}_{N}$ such that

$$
\begin{aligned}
& \left|\left\langle\phi_{k}^{u_{0}}, B \phi_{j}^{u_{0}}\right\rangle_{L^{2}}\right| \geq \widehat{C}_{N} \frac{1}{k^{3}}-\left|\frac{u_{0}}{\lambda_{j}-\lambda_{k}^{u_{0}}}\left\langle M_{k} B \phi_{k},\left(B+\widetilde{B}_{k}\right) \phi_{j}\right\rangle_{L^{2}}\right| \\
& -\left|\frac{u_{0}}{\lambda_{k}-\lambda_{j}^{u_{0}}}\left\langle\left(B+\widetilde{B}_{j}\right) \phi_{k}, M_{j} B \phi_{j}\right\rangle_{L^{2}}\right|-\left|\frac{u_{0}^{2}}{\lambda_{k}-\lambda_{j}^{u_{0}}}\left\langle\phi_{k}, L_{k, j} \phi_{j}\right\rangle_{L^{2}}\right| .
\end{aligned}
$$

2) Features of the operators $M_{k}, \widetilde{B}_{k}$ and $L_{k, j}$. Each $M_{k}$ for $k \in \mathbb{N}^{*}$ is uniformly bounded in $L\left(H_{(0)}^{2}, H_{(0)}^{2}\right)$ when $\left|u_{0}\right|$ is small enough such that

$$
\left\|u_{0}\left(\left(A-\lambda_{k}^{u_{0}}\right) P_{\phi_{k}}^{\perp}\right)^{-1} P_{\phi_{k}}^{\perp} B P_{\phi_{k}}^{\perp}\right\|_{L\left(H_{(0)}^{2}\right)}<1 .
$$

The definition of $\widetilde{B}_{k}$ implies that

$$
\widetilde{B}_{k} P_{\phi_{k}}^{\perp}=\left(\left(A-\lambda_{k}^{u_{0}}\right) P_{\phi_{k}}^{\perp}\right)^{-1} P_{\phi_{k}}^{\perp} B\left(A-\lambda_{k}^{u_{0}}\right) P_{\phi_{k}}^{\perp}-P_{\phi_{k}}^{\perp} B P_{\phi_{k}}^{\perp} .
$$

Hence, the operators $\widetilde{B}_{k}$ are uniformly bounded in $k$ in $L\left(H_{(0)}^{2} \cap \operatorname{Ran}\left(P_{\phi_{k}}^{\perp}\right), H_{(0)}^{2} \cap\right.$ $\left.\operatorname{Ran}\left(P_{\phi_{k}}^{\perp}\right)\right)$. Third, one can notice that

$$
B\left(\left(A-\lambda_{j}^{u_{0}}\right) P_{\phi_{j}}^{\perp}\right)^{-1} M_{j} B \in L\left(H_{(0)}^{2}, H_{(0)}^{2}\right)
$$

for every $j \in \mathbb{N}^{*}$. Then, for every $k \in \mathbb{N}^{*}$ and $j \leq N$,

$$
\begin{aligned}
\left(A-\lambda_{j}^{u_{0}}\right) B M_{k}\left(\left(A-\lambda_{k}^{u_{0}}\right) P_{\phi_{k}}^{\perp}\right)^{-1} P_{\phi_{k}}^{\perp} & =\left(A-\lambda_{j}^{u_{0}}\right) B\left(\left(A-\lambda_{k}^{u_{0}}\right) P_{\phi_{k}}^{\perp}\right)^{-1} \\
& \sum_{n=0}^{\infty}\left(u_{0} P_{\phi_{k}}^{\perp} B\left(\left(A-\lambda_{k}^{u_{0}}\right) P_{\phi_{k}}^{\perp}\right)^{-1}\right)^{n} P_{\phi_{k}}^{\perp} \\
& =\left(A-\lambda_{j}^{u_{0}}\right)\left(\left(A-\lambda_{k}^{u_{0}}\right) P_{\phi_{k}}^{\perp}\right)^{-1} P_{\phi_{k}}^{\perp}\left(\widetilde{B}_{k}+B\right) \widetilde{M}_{k}
\end{aligned}
$$


with

$$
\widetilde{M}_{k}:=\sum_{n=0}^{\infty}\left(u_{0} P_{\phi_{k}}^{\perp} B\left(\left(A-\lambda_{k}^{u_{0}}\right) P_{\phi_{k}}^{\perp}\right)^{-1}\right)^{n} P_{\phi_{k}}^{\perp} .
$$

Finally, the operators $\widetilde{M}_{k}$ are uniformly bounded in $L\left(H_{(0)}^{2}, H_{(0)}^{2}\right)$ as $M_{k}$. Hence $L_{k, j}$ are uniformly bounded in $L\left(H_{(0)}^{2}, H_{(0)}^{2}\right)$.

Let $\left(F_{l}\right)_{l \in \mathbb{N}^{*}}$ be an infinite uniformly bounded family of operators in $L\left(H_{(0)}^{2}, H_{(0)}^{2}\right)$. For every $l, j \in \mathbb{N}^{*}$, there exists $c_{l, j}>0$ such that

$$
\sum_{k=1}^{\infty}\left|k^{2}\left\langle\phi_{k}, F_{l} \phi_{j}\right\rangle_{L^{2}}\right|^{2}<\infty, \quad \Longrightarrow \quad\left|\left\langle\phi_{k}, F_{l} \phi_{j}\right\rangle_{L^{2}}\right| \leq \frac{c_{l, j}}{k^{2}}
$$

for every $k \in \mathbb{N}^{*}$. Now, the constant $c_{l, j}$ can be assumed uniformly bounded in $l$ since, for every $k, j \in \mathbb{N}^{*}$,

$$
\sup _{l \in \mathbb{N}^{*}}\left|k^{2}\left\langle\phi_{k}, F_{l} \phi_{j}\right\rangle_{L^{2}}\right|^{2} \leq \sup _{l \in \mathbb{N}^{*}} \sum_{m \in \mathbb{N}^{*}}\left|m^{2}\left\langle\phi_{m}, F_{l} \phi_{j}\right\rangle_{L^{2}}\right|^{2} \leq \sup _{l \in \mathbb{N}^{*}}\left\|F_{l} \phi_{j}\right\|_{(2)}^{2}<\infty .
$$

Thus, for every infinite uniformly bounded family of operators $\left(F_{l}\right)_{l \in \mathbb{N}^{*}}$ in $L\left(H_{(0)}^{2}, H_{(0)}^{2}\right)$ and for every $j \in \mathbb{N}^{*}$, there exists a constant $c_{j}$ such that

$$
\left|\left\langle\phi_{k}, F_{l} \phi_{j}\right\rangle_{L^{2}}\right| \leq \frac{c_{j}}{k^{2}}, \quad \forall k, l \in \mathbb{N}^{*} .
$$

3) Conclusion. We know that $\left|\lambda_{j}-\lambda_{k}^{u_{0}}\right|^{-1}$ and $\left|\lambda_{k}-\lambda_{j}^{u_{0}}\right|^{-1}$ asymptotically behave as $k^{-2}$ thanks to Lemma B.4. From the previous point, the families of operators $\left(B M_{k}\left(B+\widetilde{B}_{k}\right)\right)_{k \in \mathbb{N}^{*}},\left(L_{k, j}\right)_{k \in \mathbb{N}^{*}}$ are uniformly bounded in $L\left(H_{(0)}^{2}, H_{(0)}^{2}\right)$ and $B M_{j}\left(B+\widetilde{B}_{j}\right) \in L\left(H_{(0)}^{2}, H_{(0)}^{2}\right)$ for every $1 \leq j \leq N$. Hence, we use the relation (B.8) in (B.7) and there exist $C_{1}, C_{2}, C_{3}, C_{4}>0$ depending on $j \in \mathbb{N}^{*}$ such that, for $\left|u_{0}\right|$ small enough and $k \in \mathbb{N}^{*}$ large enough,

$$
\begin{aligned}
\left|\left\langle\phi_{k}^{u_{0}}, B \phi_{j}^{u_{0}}\right\rangle_{L^{2}}\right| \geq & \widehat{C}_{N} \frac{1}{k^{3}}-\frac{C_{1}\left|u_{0}\right|}{\left|\lambda_{j}-\lambda_{k}^{u_{0}}\right| k^{2}}-\frac{C_{2}\left|u_{0}\right|}{\left|\lambda_{k}-\lambda_{j}^{u_{0}}\right| k^{2}} \\
& -\frac{C_{3}\left|u_{0}\right|^{2}}{\left|\lambda_{k}-\lambda_{j}^{u_{0}}\right| k^{2}} \geq C_{4} \frac{1}{k^{3}} .
\end{aligned}
$$

Let $K \in \mathbb{N}^{*}$ be such that $\left|\left\langle\phi_{k}^{u_{0}}(T), B \phi_{j}^{u_{0}}(T)\right\rangle_{L^{2}}\right| \geq C_{4} \frac{1}{k^{3}}$ for every $k>K$. For $j \in \mathbb{N}^{*}$, the zeros of the analytic map $u_{0} \mapsto\left(\left|\left\langle\phi_{k}^{u_{0}}(T), B \phi_{j}^{u_{0}}(T)\right\rangle_{L^{2}}\right|\right)_{k \leq K} \in \mathbb{R}^{K}$ are discrete. Then, for $\left|u_{0}\right|$ small enough, $\left|\left\langle\phi_{k}^{u_{0}}(T), B \phi_{j}^{u_{0}}(T)\right\rangle_{L^{2}}\right| \neq 0$ for every $k \leq K$. Thus, for every $j \in \mathbb{N}^{*}$ and $\left|u_{0}\right|$ small enough, there exists $C_{j}>0$ such that $\left|\left\langle\phi_{k}^{u_{0}}(T), B \phi_{j}^{u_{0}}(T)\right\rangle_{L^{2}}\right| \geq \frac{C_{j}}{k^{3}}$ for every $k \in \mathbb{N}^{*}$. In conclusion, the claim is achieved for every $k \in \mathbb{N}^{*}$ and $j \leq N$ with $\widetilde{C}_{N}=\min \left\{C_{j}: j \leq N\right\}$.

Lemma B.6. Let B satisfy Assumptions I. There exists a neighborhood D(0) of 0 in $\mathbb{R}$ such that, for any $u_{0} \in D(0)$, there exist $C_{1}, C_{2}>0$ such that

$$
C_{1}\left(\left.\left.\sum_{j=1}^{\infty}|| \lambda_{j}^{u_{0}}\right|^{\frac{3}{2}}\left\langle\phi_{j}^{u_{0}}, \cdot\right\rangle_{L^{2}}\right|^{2}\right)^{\frac{1}{2}} \leq\|\cdot\|_{(3)} \leq C_{2}\left(\left.\left.\sum_{j=1}^{\infty}|| \lambda_{j}^{u_{0}}\right|^{\frac{3}{2}}\left\langle\phi_{j}^{u_{0}}, \cdot\right\rangle_{L^{2}}\right|^{2}\right)^{\frac{1}{2}} .
$$

Proof. Let $D(0)$ be the neighborhood provided by Lemma B.4. For $\left|u_{0}\right|$ small enough, we prove that there exist $C_{1}>0$ such that $\left\|\left|A+u_{0} B\right|^{\frac{s}{2}} \psi\right\|_{L^{2}} \leq$ 
$C_{1}\left\||A|^{\frac{s}{2}} \psi\right\|_{L^{2}}$ for $s=3$. We start with $s=4$ and we recall that $B \in L\left(H_{(0)}^{2}\right)$ thanks to Remark 2.1. For any $\psi \in H_{(0)}^{4}$, there exists $C_{2}>0$ such that

$$
\begin{aligned}
\left\|\left(A+u_{0} B\right)^{2} \psi\right\|_{L^{2}} \leq & \left\|A^{2} \psi\right\|_{L^{2}}+\left|u_{0}\right|^{2}\left\|B^{2} \psi\right\|_{L^{2}} \\
& +\left|u_{0}\right|\|A \psi\|_{L^{2}}\left(\|B\|\left\|_{(2)}+\right\| B \|\right) \leq C_{2}\left\||A|^{2} \psi\right\|_{L^{2}} .
\end{aligned}
$$

Classical interpolation arguments (see for instance the proof of $[\mathbf{8}$, Lemma 1]) imply the validity of the relation also for $s=3$. There exists $C>0$ such that

$$
\|\psi\|_{\widetilde{H}_{(0)}^{3}}=\left\|\left|A+u_{0} B\right|^{\frac{3}{2}} \psi\right\|_{L^{2}} \leq C\left\||A|^{\frac{3}{2}} \psi\right\|_{L^{2}}=C\|\psi\|_{H_{(0)}^{3}}
$$

for every $\psi \in H_{(0)}^{3}$. Now, $H_{(0)}^{2}=D(|A|)=D\left(\left|A+u_{0} B\right|\right)=\widetilde{H}_{(0)}^{2}$ and $B: H_{(0)}^{2} \longrightarrow$ $H_{(0)}^{2}$. The arguments of Remark 2.1 imply that $B \in L\left(\widetilde{H}_{(0)}^{2}\right)$ and the opposite inequality follows as above from the decomposition $A=\left(A+u_{0} B\right)-u_{0} B$.

Remark B.7. Let $B$ satisfy Assumptions I. The techniques of the proof of Lemma B.6 also allow to prove that, for $s \in(0,3)$, there exists a neighborhood $D(0)$ of 0 in $\mathbb{R}$ such that $\left(\sum_{j=1}^{\infty}\left|\left(\lambda_{j}^{u_{0}}\right)^{\frac{s}{2}}\left\langle\phi_{j}^{u_{0}}, \cdot\right\rangle_{L^{2}}\right|^{2}\right)^{\frac{1}{2}} \asymp\|\cdot\|_{(s)}$ for any $u_{0} \in D(0)$.

Lemma B.8. Let B satisfy Assumptions I and $N \in \mathbb{N}^{*}$. Let $\epsilon>0$ small enough and $I^{N}$ be the set defined in (1.4). There exists a $D_{\epsilon} \subset \mathbb{R} \backslash\{0\}$ such that, for each $u_{0} \in D_{\epsilon}$,

$$
\inf _{\substack{(j, k),(n, m) \in I^{N} \\(j, k) \neq(n, m)}}\left|\lambda_{j}^{u_{0}}-\lambda_{k}^{u_{0}}-\lambda_{n}^{u_{0}}+\lambda_{m}^{u_{0}}\right|>\epsilon .
$$

Moreover, for every $\delta>0$ small there exists $\epsilon>0$ such that $\operatorname{dist}\left(D_{\epsilon}, 0\right)<\delta$.

Proof. Let us consider the neighborhood $D(0)$ provided by Lemma B.3. The maps $u \mapsto \lambda_{j}^{u}-\lambda_{k}^{u}-\lambda_{n}^{u}+\lambda_{m}^{u}$ are analytic for each $j, k, n, m \in \mathbb{N}^{*}$ and $u \in D(0)$. The number of elements such that

$$
\lambda_{j}-\lambda_{k}-\lambda_{n}+\lambda_{m}=0, \quad \forall j, n, k, m \in \mathbb{N}^{*}, k, m \leq N
$$

is finite. Indeed $\lambda_{k}=k^{2} \pi^{2}$ and (B.10) corresponds to $j^{2}-k^{2}=n^{2}-m^{2}$. We have $\left|j^{2}-n^{2}\right|=\left|k^{2}-m^{2}\right| \leq N^{2}-1$, which is satisfied for a finite number of elements. Thus, for $I^{N}$ (defined in (1.4)), the following set is finite

$$
R:=\left\{((j, k),(n, m)) \in\left(I^{N}\right)^{2}:(j, k) \neq(n, m) ; \lambda_{j}-\lambda_{k}-\lambda_{n}+\lambda_{m}=0\right\} .
$$

1) Let $((j, k),(n, m)) \in R$, the set $V_{(j, k, n, m)}=\left\{u \in D \mid \lambda_{j}^{u}-\lambda_{k}^{u}-\lambda_{n}^{u}+\lambda_{m}^{u}=0\right\}$ is a discrete subset of $D(0)$ or equal to $D(0)$. Thanks to the relation (B.2),

$$
\begin{aligned}
\lambda_{j}^{u}-\lambda_{k}^{u}-\lambda_{n}^{u}+\lambda_{m}^{u}= & \left|a_{j}\right|^{2} \lambda_{j}+u\left|a_{j}\right|^{2} B_{j, j}+\lambda_{j}^{u}\left\|\eta_{j}\right\|_{L^{2}}^{2} \\
& +u \overline{a_{j}}\left\langle P_{\phi_{j}}^{\perp} B \phi_{j}, \eta_{j}\right\rangle_{L^{2}}-\left|a_{k}\right|^{2} \lambda_{k}-u\left|a_{k}\right|^{2} B_{k, k}-\lambda_{k}^{u}\left\|\eta_{k}\right\|_{L^{2}}^{2} \\
& -u \overline{a_{k}}\left\langle P_{\phi_{k}}^{\perp} B \phi_{k}, \eta_{k}\right\rangle_{L^{2}}-\left|a_{n}\right|^{2} \lambda_{n}-u\left|a_{n}\right|^{2} B_{n, n}-\lambda_{n}^{u}\left\|\eta_{n}\right\|_{L^{2}}^{2} \\
& -u \overline{a_{n}}\left\langle P_{\phi_{n}}^{\perp} B \phi_{n}, \eta_{n}\right\rangle_{L^{2}}+\left|a_{m}\right|^{2} \lambda_{m}+u\left|a_{m}\right|^{2} B_{m, m} \\
& +\lambda_{m}^{u}\left\|\eta_{m}\right\|_{L^{2}}^{2}+u \overline{a_{m}}\left\langle P_{\phi_{m}}^{\perp} B \phi_{m}, \eta_{m}\right\rangle_{L^{2}},
\end{aligned}
$$

which implies

$$
\begin{aligned}
\lambda_{j}^{u}-\lambda_{k}^{u}-\lambda_{n}^{u}+\lambda_{m}^{u}= & \left|a_{j}\right|^{2} \lambda_{j}-\left|a_{k}\right|^{2} \lambda_{k}-\left|a_{n}\right|^{2} \lambda_{n}+\left|a_{m}\right|^{2} \lambda_{m} \\
& +\left(\left|a_{j}\right|^{2} B_{j, j}-\left|a_{k}\right|^{2} B_{k, k}\right. \\
& \left.-\left|a_{n}\right|^{2} B_{n, n}+\left|a_{m}\right|^{2} B_{m, m}\right) u+o(u) .
\end{aligned}
$$


For $|u|$ small enough, thanks to $\lim _{|u| \rightarrow 0}\left|a_{j}\right|^{2}=1$ and to the third point of Assumptions I, each map

$$
u \mapsto \lambda_{j}^{u}-\lambda_{k}^{u}-\lambda_{n}^{u}+\lambda_{m}^{u}
$$

can not be constantly equal to 0 . Then, $V_{(j, k, n, m)}$ is discrete and $V=\{u \in$ $\left.D \mid \exists(j, k, n, m) \in R: \lambda_{j}^{u}-\lambda_{k}^{u}-\lambda_{n}^{u}+\lambda_{m}^{u}=0\right\}$ is a discrete subset of $D(0)$. As $R$ is a finite set

$$
\widetilde{U}_{\epsilon}:=\left\{u \in D: \forall(j, k, n, m) \in R|| \lambda_{j}^{u}-\lambda_{k}^{u}-\lambda_{n}^{u}+\lambda_{m}^{u} \mid \geq \epsilon\right\}
$$

has positive measure for $\epsilon>0$ small enough. Moreover, for any $\delta>0$ small, there exists $\epsilon_{0}>0$ such that $\operatorname{dist}\left(0, \widetilde{U}_{\epsilon_{0}}\right)<\delta$.

2) Let $((j, k),(n, m)) \in\left(I^{N}\right)^{2} \backslash R$ be different numbers. We know that

$$
\left|\lambda_{j}^{0}-\lambda_{k}^{0}-\lambda_{n}^{0}+\lambda_{m}^{0}\right|=\pi^{2}\left|j^{2}-k^{2}-n^{2}+m^{2}\right|>\pi^{2} .
$$

First, thanks to (B.2), we have $\lambda_{j}^{u} \leq\left|a_{j}\right|^{2} \lambda_{j}+|u| C_{1}$ and $\lambda_{j}^{u} \geq\left|a_{j}\right|^{2} \lambda_{j}-|u| C_{2}$ for suitable constants $C_{1}, C_{2}>0$ non depending on the index $j$. Thus

$$
\left|\lambda_{j}^{u}-\lambda_{k}^{u}-\lambda_{n}^{u}+\lambda_{m}^{u}\right| \geq\left.|| a_{j}\right|^{2} \lambda_{j}-\left|a_{k}\right|^{2} \lambda_{k}-\left|a_{n}\right|^{2} \lambda_{n}+\left|a_{m}\right|^{2} \lambda_{m}|-| u \mid\left(2 C_{1}+2 C_{2}\right) .
$$

Now, $\lim _{k \rightarrow \infty}\left|a_{k}\right|^{2}=1$. For any $u$ in $D(0)$ and $\epsilon$ small enough, there exists $M_{\epsilon} \in \mathbb{N}^{*}$ such that, for every $((j, k),(n, m)) \in R^{C}:=\left(I^{N}\right)^{2} \backslash R$ and $j, k, n, m \geq M_{\epsilon}$,

$$
\left.|| a_{j}\right|^{2} \lambda_{j}-\left|a_{k}\right|^{2} \lambda_{k}-\left|a_{n}\right|^{2} \lambda_{n}+\left|a_{m}\right|^{2} \lambda_{m} \mid \geq \pi^{2}-\epsilon .
$$

However $\lim _{|u| \rightarrow 0}\left|a_{k}\right|^{2}=1$ uniformly in $k$ thanks to (B.3) and then there exists a neighborhood $W_{\epsilon} \subseteq D(0)$ such that, for each $u \in W_{\epsilon}$, it follows

$$
\left.|| a_{j}\right|^{2} \lambda_{j}-\left|a_{k}\right|^{2} \lambda_{k}-\left|a_{n}\right|^{2} \lambda_{n}+\left|a_{m}\right|^{2} \lambda_{m} \mid \geq \pi^{2}-\epsilon
$$

for every $((j, k),(n, m)) \in R^{C}$ and $1 \leq j, k, n, m<M_{\epsilon}$. Thus, for each $u \in W_{\epsilon}$ and $((j, k),(n, m)) \in R^{C}$ such that $(j, k) \neq(n, m)$, we have $\left|\lambda_{j}^{u}-\lambda_{k}^{u}-\lambda_{n}^{u}+\lambda_{m}^{u}\right| \geq \pi^{2}-\epsilon$.

3) The proof is achieved since, for $\epsilon_{1}>0$ small enough, $\widetilde{U}_{\epsilon_{1}} \cap W_{\epsilon}$ is a non-zero measure subset of $D(0)$. For any $u \in \widetilde{U}_{\epsilon_{1}} \cap W_{\epsilon}$ and for any $((j, k),(n, m)) \in\left(I^{N}\right)^{2}$ such that $(j, k) \neq(n, m)$, we have $\left|\lambda_{j}^{u}-\lambda_{k}^{u}-\lambda_{n}^{u}+\lambda_{m}^{u}\right| \geq \min \left\{\pi^{2}-\epsilon, \epsilon_{1}\right\}$.

Remark B.9. Let $B$ satisfy Assumptions I. By using the techniques of the proofs of Lemma B.5 and Lemma B.8, one can ensure the existence of a neighborhood $D_{1}$ of $u_{0}$ in $\mathbb{R}$ and $D_{2}$, a countable subset of $\mathbb{R}$ such that, for any $u_{0} \in D(0):=\left(D_{1} \backslash D_{2}\right) \backslash\{0\}$, we have:

(1) For every $N \in \mathbb{N}^{*},(j, k),(n, m) \in I^{N}$ (see (1.4)) such that $(j, k)>(n, m)$, there holds $\lambda_{j}^{u_{0}}-\lambda_{k}^{u_{0}}-\lambda_{n}^{u_{0}}+\lambda_{m}^{u_{0}} \neq 0$.

(2) $B_{j, k}^{u_{0}}=\left\langle\phi_{j}^{u_{0}}(T), B \phi_{k}^{u_{0}}(T)\right\rangle_{L^{2}} \neq 0$ for every $j, k \in \mathbb{N}^{*}$.

(3) Let $T>0$ and $\epsilon_{0}>0$. For $\left|u_{0}\right|$ small enough, the neighborhood $O_{\epsilon_{0}, T}^{u_{0}}$ (defined in (3.6)) contains $O_{\epsilon, T}$ (defined in (3.1)) for $\epsilon>0$ sufficiently small.

\section{References}

[1] S. A. Avdonin, S. A. Ivanov, Families of exponentials, Cambridge University Press, 1995.

[2] J. M. Ball, J. E. Marsden, M. Slemrod, Controllability for distributed bilinear systems, SIAM J. Control Optim. 20, no.4 (1982), 575-597.

[3] K. Beauchard, Local controllability of a 1-D Schrödinger equation, J. Math. Pures Appl. 84, no.7 (2005), 851-956.

[4] K. Beauchard, C. Laurent, Local controllability of 1D linear and nonlinear Schrödinger equations with bilinear control, J. Math. Pures Appl. 94, no.5 (2010), 520-554. 
[5] U. Boscain, M. Caponigro, T. Chambrion, M. Sigalotti, A weak spectral condition for the controllability of the bilinear Schrödinger equation with application to the control of a rotating planar molecule, Comm. Math. Phys. 311, no.2 (2012), 423-455.

[6] U. Boscain, J. P. Gauthier, F. Rossi, M. Sigalotti, Approximate controllability, exact controllability, and conical eigenvalue intersections for quantum mechanical systems, Comm. Math. Phys. 333, no.3 (2015), 1225-1239.

[7] U. V. Boscain, F. Chittaro, P. Mason, M. Sigalotti, Adiabatic control of the Schrödinger equation via conical intersections of the eigenvalues, IEEE Trans. Automat. Control 57, no.8 (2012), 1970-1983.

[8] N. Boussaïd, M. Caponigro, T. Chambrion, Weakly coupled systems in quantum control, IEEE Trans. Automat. Control 58, no.9 (2013), 2205-2216.

[9] T. Chambrion, Periodic excitations of bilinear quantum systems, Automatica J. IFAC 48, no.9 (2012), 2040-2046.

[10] T. Chambrion, P. Mason, M. Sigalotti, U. Boscain, Controllability of the discrete-spectrum Schrödinger equation driven by an external field, Ann. Inst. H. Poincaré Anal. Non Linéaire 26, no.1 (2009), 329-349.

[11] T. Kato, Integration of the equation of evolution in a Banach space, J. Math. Soc. Japan 5 (1953), 208-234.

[12] T. Kato, Perturbation theory for linear operators, Springer-Verlag, 1995.

[13] V. Komornik, P. Loreti, Fourier series in control theory, Springer-Verlag, 2005.

[14] M. Mirrahimi, Lyapunov control of a quantum particle in a decaying potential, Ann. Inst. H. Poincaré Anal. Non Linéaire 26, no.5 (2009), 1743-1765.

[15] M. Morancey, Simultaneous local exact controllability of 1D bilinear Schrödinger equations, Ann. Inst. H. Poincaré Anal. Non Linéaire 31, no.3 (2014), 501-529.

[16] M. Morancey, V. Nersesyan, Simultaneous global exact controllability of an arbitrary number of 1D bilinear Schrödinger equations, J. Math. Pures Appl. 103, no.1 (2015), 228-254.

[17] V. Nersesyan, Global approximate controllability for Schrödinger equation in higher Sobolev norms and applications, Ann. Inst. H. Poincaré Anal. Non Linéaire 27, no.3 (2010), 901915.

[18] Y. L. Sachkov, Controllability of invariant systems on Lie groups and homogeneous spaces, J. Math. Sci. (New York) 100, no.4 (2000), 2355-2427.

Institut Fourier, Université Grenoble Alpes, Gières, MO 38610, France

Email address: alessandro.duca@univ-grenoble-alpes.fr

$U R L$ : http://www-fourier.univ-grenoble-alpes.fr/ ducaal/ 